\title{
Delay activity of saccade-related neurons in the caudal dentate nucleus of the macaque cerebellum
}

\author{
Robin C. Ashmore ${ }^{1}$ and Marc A. Sommer ${ }^{1,2}$ \\ ${ }^{1}$ Department of Neuroscience and the Center for the Neural Basis of Cognition, University of Pittsburgh, Pittsburgh, \\ Pennsylvania; and ${ }^{2}$ Department of Biomedical Engineering, the Center for Cognitive Neuroscience, and the Duke Institute for \\ Brain Sciences, Duke University, Durham, North Carolina
}

Submitted 5 October 2011; accepted in final form 28 January 2013

\begin{abstract}
Ashmore RC, Sommer MA. Delay activity of saccade-related neurons in the caudal dentate nucleus of the macaque cerebellum. $J$ Neurophysiol 109: 2129-2144, 2013. First published January 30, 2013; doi:10.1152/jn.00906.2011. - The caudal dentate nucleus (DN) in lateral cerebellum is connected with two visual/oculomotor areas of the cerebrum: the frontal eye field and lateral intraparietal cortex. Many neurons in frontal eye field and lateral intraparietal cortex produce "delay activity" between stimulus and response that correlates with processes such as motor planning. Our hypothesis was that caudal DN neurons would have prominent delay activity as well. From lesion studies, we predicted that this activity would be related to self-timing, i.e., the triggering of saccades based on the internal monitoring of time. We recorded from neurons in the caudal DN of monkeys (Macaca mulatta) that made delayed saccades with or without a self-timing requirement. Most $(84 \%)$ of the caudal DN neurons had delay activity. These neurons conveyed at least three types of information. First, their activity was often correlated, trial by trial, with saccade initiation. Correlations were found more frequently in a task that required self-timing of saccades (53\% of neurons) than in a task that did not (27\% of neurons). Second, the delay activity was often tuned for saccade direction (in $65 \%$ of neurons). This tuning emerged continuously during a trial. Third, the time course of delay activity associated with self-timed saccades differed significantly from that associated with visually guided saccades (in $71 \%$ of neurons). A minority of neurons had sensory-related activity. None had presaccadic bursts, in contrast to DN neurons recorded more rostrally. We conclude that caudal DN neurons convey saccade-related delay activity that may contribute to the motor preparation of when and where to move.
\end{abstract}

motor preparation; timing; vision; recurrent loops; macaque

THE CEREBRAL CORTEX OF ALL vertebrates with complex telencephela is linked via recurrent circuits with three deeper, evolutionarily conserved structures: the basal ganglia, brain stem, and cerebellum. An expansive literature has detailed the activity of neurons in cortical-basal ganglia loops in mammals (Alexander et al. 1986; McHaffie et al. 2005; Middleton and Strick 2000; Parent and Hazrati 1995) and birds (Bottjer and Altenau 2010; Farries 2004; Perkel 2004), and several studies have examined neuronal activity in noncatecholaminergic, cortical-brain stem loops (e.g., in primates: Crapse and Sommer 2009; Helminski and Segraves 2003; Segraves and Goldberg 1987; Sommer and Wurtz 2000, 2001, 2004; in birds: Ashmore et al. 2008). In contrast, little is known about neuronal activity in cortical-cerebellar loops.

Address for reprint requests and other correspondence: R. C. Ashmore, Keystone Bldg, 3520 Fifth Ave., Suite 300, Pittsburgh, PA 15213 (e-mail: rca@cnbc.cmu.edu).
The organization of cortical-cerebellar loops has been established in nonhuman primates anatomically (Kelly and Strick 2003; Lynch et al. 1994; Middleton and Strick 1994, 2000; Wiesendanger and Wiesendanger 1985; Yamamoto et al. 1992) and electrophysiologically in anesthetized preparations (Sasaki et al. 1976, 1977, 1979). Evidence for the loops in humans has been provided by functional connectivity MRI (Allen et al. 2005; Habas et al. 2009; Krienen and Buckner 2009). The classical view is that cortico-cerebellar loops contribute to motor control (Evarts and Thach 1969; Ito 2002; Stein 1986). An emerging hypothesis, based on connectivity and human lesion results, is that the specific set of loops that link lateral cerebellum with frontal cortex contribute to nonmotor functions, such as working memory, attention, verbal fluency, and action planning (Bellebaum and Daum 2007; Ito 2002; Lagarde et al. 2009; Middleton and Strick 2000; Schmahmann 1997; Strick et al. 2009; Thach 2007). Physiological evidence for this nonmotor hypothesis has been provided by functional MRI results in humans (Habas 2010; Kim et al. 1994; Küper et al. 2011; Salmi et al. 2010) and by neuronal recording studies in monkeys that reported arm-related activity in the dentate nucleus (DN) of the lateral cerebellum that depended on behavioral context (Chapman et al. 1986; Middleton and Strick 2000; Strick 1983; Thach 1978).

In behaving monkeys, the DN makes functional connections with cerebral cortex, as indicated by short-latency, orthodromic activation of motor cortex neurons following DN stimulation (Holdefer et al. 2000). Lynch et al. (1994) discovered that a specific zone of DN is part of a loop with an oculomotor region of frontal cortex, the frontal eye field (FEF). Neurons in the caudal pole of DN send a disynaptic pathway to the FEF via the ventrolateral (VL) thalamic nucleus (Lynch et al. 1994). The loop is closed by FEF neurons that project to dorsal, medial, and dorsolateral pontine nuclei in regions that overlap with pontine projections to the lateral cerebellum (Huerta et al. 1986; Leichnetz 1989; Schmahmann and Pandya 1997; Xiong et al. 2002) that in turn synapse onto Purkinje cells innervating the caudal DN (Kelly and Strick 2003; Xiong et al. 2002). More recently, it was demonstrated (Prevosto et al. 2010) that the caudal pole of the DN also sends a disynaptic pathway via thalamus to the ventral portion of lateral intraparietal (LIP) cortex, another well-known visuosaccadic cerebral cortical area. Additionally, the caudal DN projects directly to the superior colliculus (SC) (May et al. 1990), where, according to anatomical criteria, terminations seem to contact saccaderelated burst neurons (Prevosto et al. 2007). 
Taken together, the anatomical evidence implicates the caudal pole of the DN as a specialized oculomotor domain (Prevosto et al. 2010). Its connections suggest a role that could be different from that of a more medial cerebellar area, the caudal fastigial nucleus, which contributes to the fine control of saccade dynamics and endpoint (e.g., Fuchs et al. 2010; Goffart et al. 2003; Robinson and Fuchs 2001). The FEF, LIP, and the $\mathrm{SC}$ are strongly implicated in processes that accompany saccades, such as discriminating visual saccade targets from distractors (Carello and Krauzlis 2004; Nummela and Krauzlis 2010; Schall and Thompson 1999; Thomas and Paré 2007) and encoding the saccade's vector and time of initiation (Bruce et al. 1985; Colby et al. 1996; Dias and Segraves 1999; Munoz and Wurtz 1995; Schall and Hanes 1998; Sommer and Tehovnik 1997; Wurtz et al. 2001). These preparatory processes occur after stimulus onset and before saccade initiation, so they are often studied at the single-neuron level using tasks that impose a delay between stimulus and response. A hallmark of many neurons in the FEF, LIP, and SC is that they exhibit prominent "delay activity" in such delayed response tasks. Our overall hypothesis was that neurons in caudal DN produce delay activity as well, and that this activity is related to preparatory processes for saccades.

One preparatory process that DN neurons might encode is the timing of voluntary behavior, or "self-timing". In a study of self-timed finger tapping in patients with focal cerebellar lesions, Ivry et al. (1988) found that patients with lateral cerebellar lesions behaved as if a central clock process was impaired. When the patients made an error in the tapping rhythm, they continued as if unaware of the error. In contrast, patients with medial cerebellar lesions compensated for their tapping errors to recover the original rhythm (for more on this and related work, see Ivry and Keele 1989; Spencer et al. 2003; and reviews, Ivry 1996; Mauk and Buonomano 2004). In the cerebral targets of caudal DN, neural activity hypothesized to represent temporal aspects of sensation or movement has been found in both FEF (Li and Lisberger 2011) and LIP (Janssen and Shadlen 2005; Leon and Shadlen 2003; Onoe et al. 2001). In the thalamus, Tanaka $(2006,2007)$ showed that regions implicated in the DN-frontal cortex pathway play a role in triggering of saccades based on the internal monitoring of time. The hallmark of timing-related neuronal activity in the thalamus was a prolonged increase or decrease ("ramping") of firing rate during self-monitored waiting periods before saccades (Tanaka 2007).

Neither delay activity nor signals related to self-timing have been found previously in the DN. Some DN neurons have been shown to fire in relation to eye movements (Gardner and Fuchs 1975; Thach 1975), but their activity primarily consisted of a burst that started $30 \mathrm{~ms}$ or less before saccade initiation (Chubb and Fuchs 1982; Gardner and Fuchs 1975; Hepp et al. 1982; MacKay 1988) and ended in precise correlation with saccade termination (Gardner and Fuchs 1975; Hepp et al. 1982). The neurons were distributed broadly in the rostrocaudal dimension across the ventral half of the nucleus (MacKay 1988; Thach 1978; van Kan et al. 1993).

We tested the hypothesis that neurons in the caudal pole of the DN produce delay activity, and we made the specific prediction that such activity is related to self-timing. We recorded from single neurons in the caudal pole of $\mathrm{DN}$ in monkeys that performed a battery of delayed saccade tasks, some of which included a self-timing requirement. Supporting our hypothesis, we found that most neurons in the caudal DN were highly active during the tasks, but lacked the discrete saccade-related bursts typical of neurons elsewhere in the nucleus (Chubb and Fuchs 1982; Gardner and Fuchs 1975; Hepp et al. 1982; MacKay 1988). Instead, neurons in the caudal DN produced vigorous, prolonged delay activity that was related to saccade initiation, conveyed information about saccade direction, and differed between tasks that did or did not require self-timing.

\section{METHODS}

Experiments were performed on two male rhesus macaques: "R" and "F". Under anesthesia in aseptic conditions, the monkeys were implanted with scleral search coils, head post holders, and a recording chamber targeting the cerebellum. The chamber was angled $30 \mathrm{de}-$ grees from vertical in the sagittal plane (top of chamber posterior to bottom of chamber) and offset laterally to the left of midline by $3 \mathrm{~mm}$. All procedures were performed at the University of Pittsburgh and approved by the university's Institutional Animal Care and Use Committee and were in accordance with the regulations detailed in the National Institutes of Health Guide for the Care and Use of Laboratory Animals.

During experiments, the monkey's head was held still while it sat in a standard primate chair (Crist Instrument) facing a monitor or back-projected screen (see below). Neuronal recordings were targeted using a grid (holes spaced $1 \mathrm{~mm}$ apart; Crist) fixed in the chamber. To enable sampling of the DN at submillimeter scales, we used grids in which holes were offset from the standard grid by $0.5 \mathrm{~mm}$, we rotated the grids at reproducible small angles in the chamber, or both. A 23-gauge guide tube was inserted through a grid hole and through the dura. To keep electrodes on straight trajectories and maximize our ability to target the same region of caudal DN repeatedly, the guide tubes were inserted $8-10 \mathrm{~mm}$ into the cerebral cortex, bringing the end of the guide tubes within $\sim 4 \mathrm{~mm}$ of the dorsal cerebellar surface. A standard grid was used to access left DN (located directly below the chamber), and an angled grid (10 degrees from vertical) provided access to the right DN.

\section{Recordings}

We recorded from single neurons with epoxylite-insulated tungsten electrodes (500-1200 k $\Omega$ at 1,000 Hz; FHC, Bowdoinham, ME) inserted through the guide tube and advanced with a motorized microdrive (Motion Group, Clovis, CA). Eye movement data were recorded from the scleral search coils using a combined amplifier and field coil driver system (Riverbend Instruments, Birmingham, AL), or from a video-based eye tracker (for 3 neurons studied in monkey "R"; ISCAN, Woburn, MA). Eye movement data were digitized and stored, along with codes representing neuronal spike times and task events, at 1-ms resolution by a computer running the REX software suite (National Eye Institute). REX was also programmed to present visual stimuli, monitor behavior, and deliver reward (drops of water). Saccade detection was performed using a threshold eye velocity over a minimum time interval ( $>15 \%$ for $10 \mathrm{~ms}$ or more). Neuronal spike times were determined with action potential waveform discrimination accomplished using a separate module, MEX, which ran on another computer. Auditory stimuli were presented with a custom MS Windows application (written for this study in $\mathrm{C}++$ and given the name "Avex"). Both MEX and Avex were linked directly to REX through digital I/O boards (National Instruments, Austin, TX). When necessary, such as to verify the presence of complex spikes in the cerebellar cortex or to distinguish single from multiple units, continuous neuronal waveform data were recorded and digitized at a sampling rate of 
$96 \mathrm{kHz}$ using the Avex computer (Fast Track Pro Audio Interface, Avid, Irwindale, CA).

Recordings were made in the lateral cerebellar cortex and deep nuclei, with a focus on the DN. Each DN was mapped extensively with recordings to determine its caudal and lateral borders $(6-7 \mathrm{~mm}$ lateral from the midline), and its location was verified by structural MRI with implanted electrodes for reference. An example magnetic resonance image from monkey " $\mathrm{R}$ " is shown in Fig. $1 A$ along with matching, scaled atlas illustrations for reference (Fig. $1 B$; Mikula et al. 2008). At the conclusion of experiments, we additionally confirmed the caudal DN recording locations histologically in monkey "F" (Fig. $1 C$, details in Histology below). During recording sessions, we could readily distinguish the $\mathrm{DN}$ from cerebellar cortex on the basis of 1) depth referenced to MRI measurements; 2) surrounding regions of white matter as evidenced by little neuronal activity; 3) complex spikes above, and depending on location, below the white matter; and 4 ) no complex spikes in the DN itself.

\section{Behavioral Tasks}

We used four tasks that required monkeys to make saccades from a central fixation spot to a cued peripheral location (Fig. 2). The tasks were presented in blocks (e.g., 30 trials of one task, followed by 30 trials of another) with peripheral cue locations pseudorandomized. To aid monkeys in distinguishing the tasks, each used a unique fixation spot color (not depicted in Fig. 2).

Visual self-timing task. The purpose of this task was to require monkeys to monitor the time that elapses after a visual event to initiate saccades successfully. The task's structure (Fig. 2, top) was similar to that used by Tanaka $(2006,2007)$. A green central fixation point appeared, and a monkey was required to foveate it for 100-400 ms (pseudorandomized). This was followed by a brief target cue in one of eight locations (along the cardinal and diagonal directions) for $\sim 110$-ms duration. There was minor variability in cue durations due to projector refresh times, as can be seen in the figures, but this was documented by a photocell on the screen and was irrelevant for this study. After the visual cue disappeared, the monkey was required to continue foveating the central point for $600 \mathrm{~ms}$ (the "delay period"). Once $600 \mathrm{~ms}$ elapsed, the monkey had to make a saccade to the target location within an 800-ms duration "acceptance" interval. Critically, monkeys were provided with no cues to inform them when the saccade was permitted; they had to accomplish the timing themselves.
If a saccade was made to the correct location during the acceptance interval, the visual cue reappeared immediately when the saccade ended. The monkey had to fixate it for $100 \mathrm{~ms}$ for successful conclusion of the trial and delivery of liquid reward. In the event of a premature saccade or any other failure to keep the fixation point foveated (within a $3 \times 3^{\circ}$ window) prior to the acceptance interval, the screen was filled by a large square covering $10-20^{\circ}$ of visual angle for $1,400 \mathrm{~ms}$. If no saccade was made during the acceptance interval, the trial was aborted. Intertrial intervals were always 1,500-2,000 ms (pseudorandomized). Trials in which a monkey's eyes left the fixation point too early, too late, or in which the target was not attained and foveated for $100 \mathrm{~ms}$ were excluded from analysis.

In the visual self-timing (VS) task, as in all of the tasks, we presented a brief auditory tone (50 $\mathrm{ms}$, behind the animal's head) and a small water reward $100 \mathrm{~ms}$ after the start of fixation. This provided a common, highly salient event at the same time early in all trials, to help maintain comparable arousal levels across tasks. In all of the tasks, liquid reward for correct saccades was at least twice the size of the fixation period reward.

Auditory self-timing task. This task required self-timing of saccades, as with the VS task, but used an auditory rather than a visual spatial cue (Fig. 2, second from top). The purpose of the auditory self-timing (AS) task was to assess whether putative self-timingrelated activity depended on vision. A monkey foveated a central, yellow fixation point, but no peripheral visual cue appeared. Instead, a complex frequency- and amplitude-modulated sound was played through one of four speakers placed around the video screen $\left(\sim 45^{\circ}\right.$ of arc from the fixation point). The direction of the speaker relative to the fixation point indicated the direction of the required saccade. We found that head-restrained monkeys cannot easily distinguish sounds that differ only on the vertical axis (which create no interaural timing difference), and, therefore, we used distinct sounds for vertical directions. After the auditory cue, the monkey had to wait through the delay period $(600 \mathrm{~ms})$ and then, without any external cue to move, make a saccade during a 2,000-ms acceptance interval. This interval was longer than in the VS task to compensate for the apparent difficulty that the monkeys experienced in making vertical, auditoryguided saccades. If a monkey made its saccade to the correct location, a visual stimulus was presented at that location immediately following the movement. The monkey had to fixate it for $100 \mathrm{~ms}$, and reward was delivered.
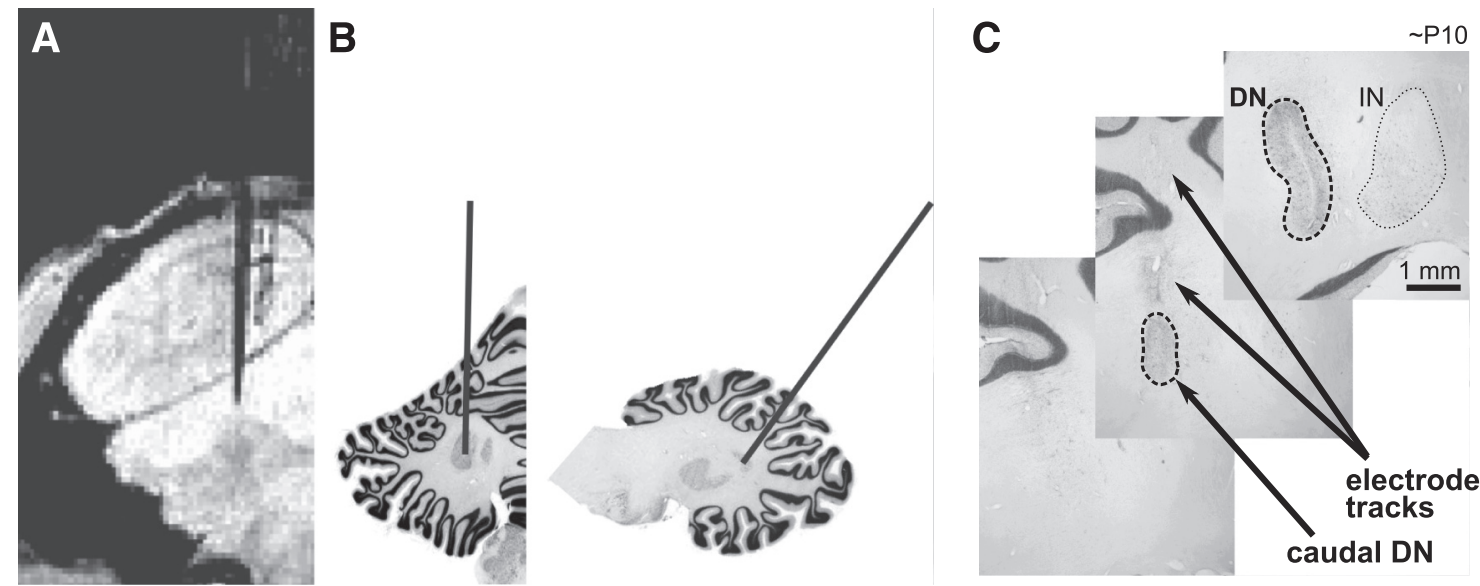

Fig. 1. Anatomical localization of caudal dentate nucleus (DN) recordings. A: example MRI section of monkey $R$ (coronal slice, left hemisphere). A temporarily implanted reference electrode was aimed at the electrophysiologically identified left DN, but positioned with its tip several millimeters above recording sites to prevent damage during the MRI procedure. Shadow tracks in the MRI (medial to reference electrode) are from exploratory electrode penetrations. $B$ : electrode paths reproduced from coronal (left) and parasagittal (right) MRI sections, reproduced onto scaled excerpts of atlas sections from http://brainmaps.org/ajaxviewer.php?datid $=3 \&$ sname $=0394$ [from Mikula et al. 2008], showing intersection of trajectory with ventral-caudal DN. $C$ : histology from monkey $F$, showing cerebellar sections $250 \mu \mathrm{m}$ apart from rostral (top) to caudal (bottom). Top: in this section, $10 \mathrm{~mm}$ posterior to the interaural line ("P10"), the DN and the interpositus nucleus (IN) are visible, both outlined for clarity. Middle: slightly more posterior, the caudal pole of the DN is visible (outlined), as well as gliosis above it from repeated electrode penetrations into it. Bottom: one more section posterior to that, the DN is not present any more. 


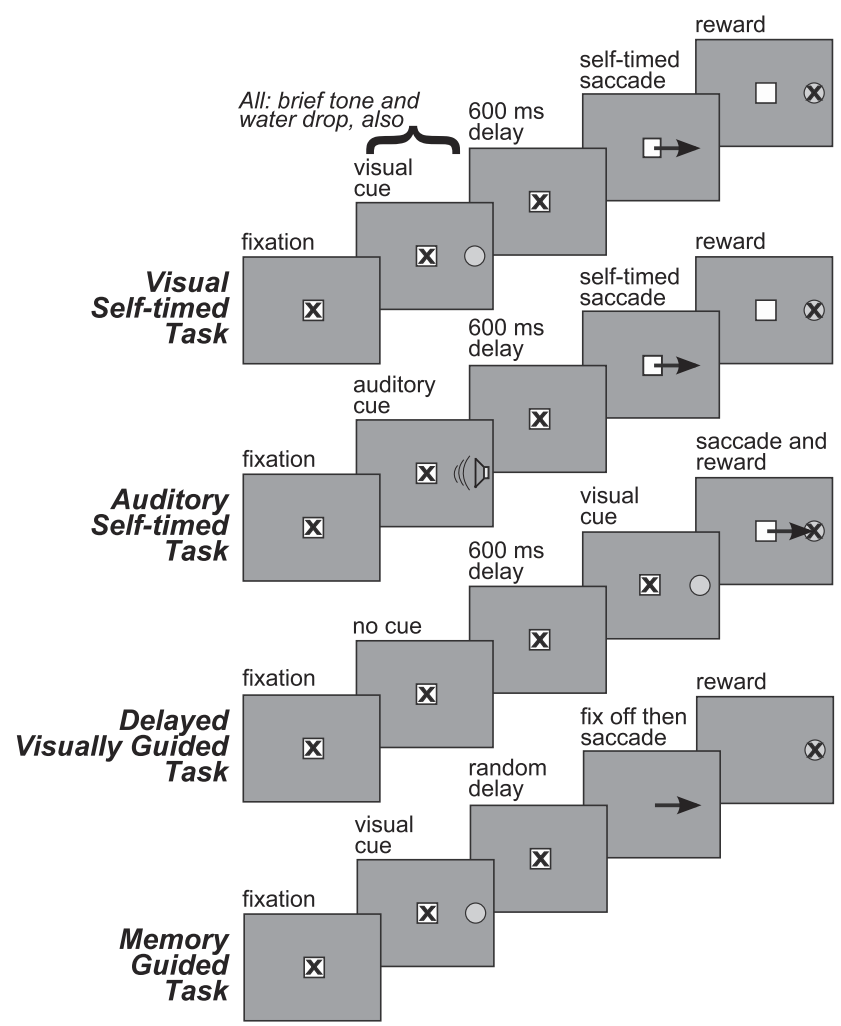

Fig. 2. Spatiotemporal design of the tasks. In all four tasks, the monkey began by fixating a central spot. To assist the monkey in distinguishing between the tasks, fixation spot colors differed by task (not shown). After a brief period of fixation, a nondirectional tone pip and a drop of water were delivered (as labeled). Arrows depict saccades, X's depict foveations, squares represent fixation spots, and circles represent visual cues for the required saccades. Intertrial intervals (not shown) were always 1,500-2,000 ms. Tasks from top to bottom: visual self-timed (VS) task: in this task, simultaneous with the tone and water drop, a visual target cue appeared briefly in 1 of 8 locations. The animal was required to initiate its saccade to the location of the remembered cue 600-1,400 ms later. Monkeys had to self-time the waiting period; there was no event that signaled when to move. Auditory self-timed (AS) task: this was identical to the VS task, except that the eventual saccade goal was cued by a directional sound. Icon represents a speaker producing an auditory stimulus to the right of the monkey. Delayed visually guided (DVG) task: in this condition, self-timing of saccades was not required. Early in a trial, the animal received the tone and water drop as usual, but no other stimuli were presented. The monkey continued fixating for $600 \mathrm{~ms}$, and then a visual target appeared, to which the monkey made a saccade after a reaction time. The fixation spot remained lit until the end of the trial. Memory-guided (MG) task: in this condition, a visual target location had to be remembered as in the VS task, but self-timing of saccades was not required. Disappearance of the fixation point after the delay period provided an explicit cue to move.

Delayed visually guided task. This task was a comparison condition for which self-timing of saccades was reduced as much as possible (Fig. 2, third from top). Self-timing was not required, and there was no fixation point disappearance [which may promote temporal anticipation as described by Tanaka (2007)]. The monkey simply made visually guided saccades after an imposed delay. The monkey foveated a blue fixation point for $100 \mathrm{~ms}$, and then, as in the other tasks, a brief nondirectional tone and small reward were delivered. After 600 $\mathrm{ms}$, a peripheral visual target appeared in one of eight possible locations (cardinal directions and diagonals). If the monkey made a saccade directly to the target within $800 \mathrm{~ms}$, the trial was correct, and reward was delivered. The fixation point remained present until the end of the trial.

Memory-guided task. This was another comparison condition for which self-timing was not required. We modified a standard memoryguided (MG) saccade task (Hikosaka and Wurtz 1983; Mays and
Sparks 1981; Tanaka 2006) to include, during early fixation, the initial nondirectional tone and small reward as in the other tasks. A purple fixation point appeared, and a monkey was required to foveate it for 100-400 ms (pseudorandomized). Then a visual cue appeared in one of eight locations (along the cardinal and diagonal directions) for $\sim 110 \mathrm{~ms}$. After the cue disappeared, the monkey was required to continue foveating the central point for a delay period of $300-600 \mathrm{~ms}$, pseudorandomized to discourage temporal anticipation. When the delay period ended, the fixation point disappeared, and the monkey had to make a saccade directly to the correct location within $800 \mathrm{~ms}$. If the monkey succeeded, the remembered cue reappeared, the monkey had to fixate it for $100 \mathrm{~ms}$, and reward was delivered.

Training on self-timing tasks. To train the monkeys, we started with the MG task, which was familiar to them. We began leaving the fixation point on throughout the trial, which forced the monkeys to try different behaviors to earn their reward. Within a few days to weeks, they learned that they had to break fixation on their own after $600 \mathrm{~ms}$ to initiate their MG saccade with no external cue. Once trained on the VS task, they were able to generalize to the auditory version of the task (the AS task).

\section{Analyses}

Our analyses focused on the main type of activity exhibited by the caudal DN neurons: modulations in firing rate during the delay period of the tasks ("delay activity").

Significance of delay activity. To evaluate overall significance of delay activity, we used two main tests. For both tests, data were segregated by neuron, task type, and saccade direction. The purpose of the first test was to determine which portions of the delay activity, if any, differed significantly from baseline activity. The second test checked the validity of the first test by analyzing delay activity independent of baseline activity (in case baseline activity itself conveyed preparatory signals that would complicate comparisons with delay activity).

For the first baseline-comparison test, the spike trains of each neuron during the 600 -ms delay period were divided into six $100-\mathrm{ms}$ bins, and the mean firing rate and standard deviation of spike counts in these bins were calculated across all trials of a specific task type and direction. These data were each compared with the mean baseline firing rate recording during the last $100 \mathrm{~ms}$ preceding target cue onset for the same trials. Comparisons were made using a standard $t$-test with a Bonferroni correction of $P<0.0083$ to account for multiple comparisons ( $n=6$ bins) in each data set. We considered a neuron to be significantly modulated, relative to baseline, for a given task and direction if any of the six bins were significantly different from the baseline period.

We also noted that many of these significantly modulated neurons showed a pronounced decay from baseline firing rate during the delay period (termed a "pause"). To quantify the pauses, we used the same baseline period (100-0 ms before target cue onset), with trials again grouped by neuron, task, and direction. We calculated a spike density function (SDF; Gaussian kernel of $20 \mathrm{~ms}$; MacPherson and Aldridge 1979) across all trials for this period. The mean rate during this period was compared with a SDF computed for the whole delay period (again computed from all trials for a given neuron, task, and direction). Neurons were considered to have a significant delay period pause for the task and direction if the SDF in the delay period decreased more than 2 standard deviations below the baseline mean for at least 50 contiguous ms.

For the second baseline-independent test, we used a bootstrap procedure that quantified whether modulations in delay activity over time differed from those that could be produced by random firing. For a given neuron, task, and direction, all trials were aligned to the start of the delay period. A single SDF was generated from the trials for $600 \mathrm{~ms}$ and divided into 60 bins. A vector of 60 numbers was generated by computing the mean spike density within each bin. We 
compared this vector to random firing patterns constructed from data from the same neuron. For each iteration of our bootstrap test, two groups of random spike trains were generated by drawing randomly (with replacement) from the trials for all directions for the task, and within each drawn trial, drawing a 1-ms point at random (with replacement). A SDF and 60-bin vector was generated for each of the two random spike train groups. One of these was arbitrarily designated the "comparison vector", and the other the "random test vector". We measured the Euclidean distance between these two and then between the observed neural data vector and the comparison vector. The process was repeated 500 times to generate a distribution of observed distances ("observed" to "comparison") and distances expected by chance ("random" to "comparison"). Using a $z$-test, we considered as significant any observed vector (representing a recorded set of trials) that had a mean distance measure $>2$ standard deviations from the distances expected by chance. In sum, delay activity modulation identified as significant by this bootstrap method was dissimilar from that expected by chance (at $P<0.0228$ ) and, therefore, changed significantly with time (e.g., in upward or downward ramps).

Neuronal-saccadic latency correlations. To determine the relationship between delay activity and behavior, we performed latency correlation tests between saccade initiation times and identifiable changes in firing rate near the end of the delay period. Many neurons exhibited consistent changes in firing rate after the 600-ms delay period, around the time of saccade initiation. Such changes included marked increases in firing rate, marked decreases, or inflections in the rate (the rate reached a peak or trough). These changes in firing rates were clear by inspection and appeared to correlate temporally with saccade initiation (see Fig. 5A). To quantify this qualitative observation, we developed an algorithm to objectively measure the occurrence and timing of these firing rate changes (slopes and inflections). The algorithm computed ascending or descending firing rate threshold crossings, or the time of peak activity, as computationally derived correlates of these firing rate changes.

The algorithm worked as follows. Trials were first separated into groups (one group $=$ all trials for one task and one direction for one neuron). Groups with less than eight trials were excluded. A starting threshold value was determined from the mean of the firing rates for all trials during the interval from 600 to $1400 \mathrm{~ms}$ after cue onset $(3.4 \times$ mean rate for ascending crossings, $0.05 \times$ mean rate for descending crossings). All trials in the group were tested to see if either threshold value was crossed during the same time interval. If fewer than one-half of the trials crossed either threshold (or at minimum, six trials), the thresholds were adjusted by $20 \%$. This adjustment was repeated up to six times until an appropriate threshold was found. For trial groups with too few trials containing any of the threshold crossings, the time of the maximum neural activity was used (the maximum activity was the threshold). By employing this method of successively increasing and decreasing thresholds, we were able to objectively capture the rate slopes and inflections that we noted on initial inspection. Matlab code for implementing the algorithm is available from the corresponding author (R. C. Ashmore).

To determine whether the algorithm-identified features in the neuronal activity were temporally related to saccade initiation, we computed the correlation between the timing of these features (the "neural latency") and saccade onset ("saccade latency", defined as the time from the start of the delay period to the time when eye speed began to exceed $15 \%$ for $10 \mathrm{~ms}$ or more). We evaluated the correlations between neuronal latency and saccadic latency using Pearson's test. Positive correlations meant that the longer the neuronal latency, the longer the saccadic latency; negative correlations meant that the longer the neuronal latency, the shorter the saccadic latency.

Direction selectivity. Delay activity for many neurons varied by direction of movement. We analyzed this direction selectivity using two approaches. In the first test, we focused on data from the VS task, for which we had the most data. We segmented each neuron's delay activity from this task into six sequential, 100-ms bins. Within each bin, we performed an ANOVA across data from the eight directions tested $(P<0.0083$ criterion to account for the six tests for each neuron). A neuron was deemed to have direction selectivity if the ANOVA was significant for any bin; hence, some of the direction selectivity we report was transient. Indeed, we found in general that direction selectivity was labile. For neurons that were directionally selective in all six bins, we observed that the directional tuning changed markedly during the first one-half of the delay period. We analyzed these rotations in directional selectivity during the delay period for that subset of neurons, and for the entire sample of neurons, using standard statistical tests, as described in RESULTS.

In the second test, we focused on the strength and fine-scale temporal development of directional selectivity using a receiver operating characteristic (ROC) bootstrap analysis (Green and Swets 1966; Horwitz and Newsome 2001; Phillips and Segraves 2010). For each neuron and task [VS, AS, or delayed visually guided (DVG)], we performed a preliminary ROC comparison between all direction pairs to identify the two directions that elicited the most dissimilar activity (designated for analysis as $\mathrm{d} 1$ and $\mathrm{d} 2$ ). Activity in the $\mathrm{d} 1$ and $\mathrm{d} 2$ directions was aligned with saccade initiation, and an ROC curve was generated every $5 \mathrm{~ms}$ over the preceding $600 \mathrm{~ms}$. The ROC curves were calculated from 10-ms segments, so that the first ROC curve used spikes from 600-590 ms before saccade onset, the second from 595-585 ms before saccade onset, and so on, for a total of 120 ROC curves for the pair of directions.

Details of the ROC curve calculations were as follows. A 10-ms span of all trials for $\mathrm{d} 1$ and the corresponding 10-ms span of all trials for $\mathrm{d} 2$ were both compared with an increasing list of 10 thresholds (from $i=1$ to 10 spikes $/ 10 \mathrm{~ms}$ bin). Ten points created a piecewise curve $P($ threshold $)$, where each point $P\left(\right.$ threshold $\left._{i}\right)$ was an $(x, y)$ value corresponding to $x=$ (proportion of $\mathrm{d} 1$ trials in which the spike count exceeded threshold $i$ ) and $y=$ (proportion of $\mathrm{d} 2$ trials in which the spike count exceeded threshold $i$ ). The area under the curve (AUC) of the resulting $P$ function was used as a measure of difference between the $\mathrm{d} 1$ and $\mathrm{d} 2$ directions. This algorithm, therefore, generated 120 AUC values, representing the observed differences in neuronal activity between the two directions, measured every $5 \mathrm{~ms}$. We tested for significance of each observed AUC value using a bootstrap method, each iteration of which followed the same process outlined above. When generating the list of ROC curves and AUC values, however, trials for the two directions were drawn randomly (with replacement) from the pool of all trials for both directions. In other words, the trials were randomly assigned to the $\mathrm{d} 1$ and $\mathrm{d} 2$ groups during each bootstrap iteration, and 120 AUC values were calculated from the randomized groups. This randomization procedure was repeated 500 times, generating a distribution of AUC values to be expected by chance. A single AUC value from the observed data was significant if it was greater than $95 \%$ of the randomly generated AUC values (i.e., $P<0.05$ ).

Activity for a given neuron was considered directionally selective if there were at least 13 significant AUC values in the array of 120 AUC values generated from the observed data. The value 13 was chosen by modeling the frequency with which $N$ bins of significant AUC values would be expected to appear in lists of 120 random AUC values, where $N$ ranged from 1 to 35 . We found that, once 13 significant bins had been identified in a neuron's activity, the probability of this occurring by chance dropped below 0.05 (precisely, $P=$ 0.0216). A neuron's latency for direction selectivity, i.e., the time at which two directions $\mathrm{d} 1$ and $\mathrm{d} 2$ could be predicted from the neuronal activity, was defined as the time of the first bin of five or more consecutive significant bins. By this definition, latency could not be measured from some neurons that were nonetheless significantly direction selective (had 13 or more significant bins).

To compare the ROC results with the ANOVA results of the first test, which analyzed only VS task data, in a second pass of analysis we repeated the ROC analysis on data solely from the VS task. For this analysis, the presence of 11 or more significant bins corresponded to the $P<0.05$ criterion level (precisely, $P=0.0388$ ). 
Task selectivity. To assess the proportion of neurons that carried information specifically related to self-timing, we compared delay activity evoked in the VS task (which required self-timing) and the DVG task (which did not) using the same bootstrap ROC method as described above for direction selectivity. AUC values were computed separately for the eight task directions, such that "up" trials for the VS task were compared with "up" trials for the DVG task, "left" with "left", and so on. Values reported are those for the pair with the largest number of significant AUC bins. We adjusted our expectancy model to account for this process of taking the maximum of eight computations and found that producing random AUC arrays with 14 significant bins corresponded to $P<0.05$ (precisely, $P=0.0219$ ).

Sensory responses. To quantify and compare possible sensory responses, we computed the mean firing rates during two epochs for the VS and AS tasks. The baseline epoch spanned 0-100 ms preceding the onset of the visual (VS) or auditory (AS) target cue, and the sensory epoch spanned 50-150 ms following the cue onset. For a given neuron, task, and direction, the firing rates in these two epochs were compared for all trials using a paired $t$-test.

\section{Histology}

To verify the site of our recordings in one animal, we made electrolytic lesions posterior and dorsal to our recording sites in both hemispheres, using cathodal $20-40 \mu \mathrm{A}$ DC for $60 \mathrm{~s}$. Then the animal was deeply anesthetized ( $25 \mathrm{mg} / \mathrm{kg}$ ketamine im), given an overdose of pentobarbital sodium (37 mg/kg ip), and perfused transcardially with $0.1 \mathrm{M}$ phosphate buffer, $10 \%$ buffered formalin, followed by $10 \%$ buffered formalin with $10 \%$ glycerol added. After the perfusion, the brain was removed and stored in 10\% buffered formalin with $20 \%$ glycerol $\left(4^{\circ} \mathrm{C}\right)$ for $5-7$ days. The cerebellum was sectioned coronally (50- $\mu \mathrm{m}$ sections), and each fourth section was stained with cresyl violet.

\section{RESULTS}

We recorded from 79 neurons in the caudal pole of DN (Fig. 1). The sample included 49 neurons from monkey $F$ (23 neurons from the left DN plus 26 neurons from the right DN) and 30 neurons from monkey $R$ (all from the left DN). The most striking characteristic of the neurons was their prominent delay activity, which spanned long periods of time leading up to movement initiation. Figure 3 shows firing patterns of three typical neurons recorded during the VS task. For all three neurons (and all neurons tested), eye position remained steady during the delay period. The first neuron (Fig. $3 A$ ) exhibited an initially high $(>100$ spikes/s) firing rate that dropped to zero during target cue presentation, turned on again at an elevated rate $(\sim 200$ spikes/s) during the self-timed waiting period, and then dropped back to zero near the time of the saccade. The second neuron (Fig. 3B) showed "upward ramping" activity. Similar to the first neuron, it stopped firing around the time of cue presentation, became active during the delay, and then ceased firing again after the saccade. Different from the first neuron, the firing rate steadily accelerated during the delay; its average activity rose in a near-linear fashion over $400 \mathrm{~ms}$ to reach a peak of $\sim 150$ spikes/s around saccade initiation. As can be seen, the steady increase occurred in individual trials as well as on average; i.e., the ramp was not an artifact of averaging staggered onset times of steady activities. The third neuron (Fig. 3C) was "downward ramping". It displayed a high firing rate at the beginning of the delay period that decreased and then ceased around the time of saccade initiation.
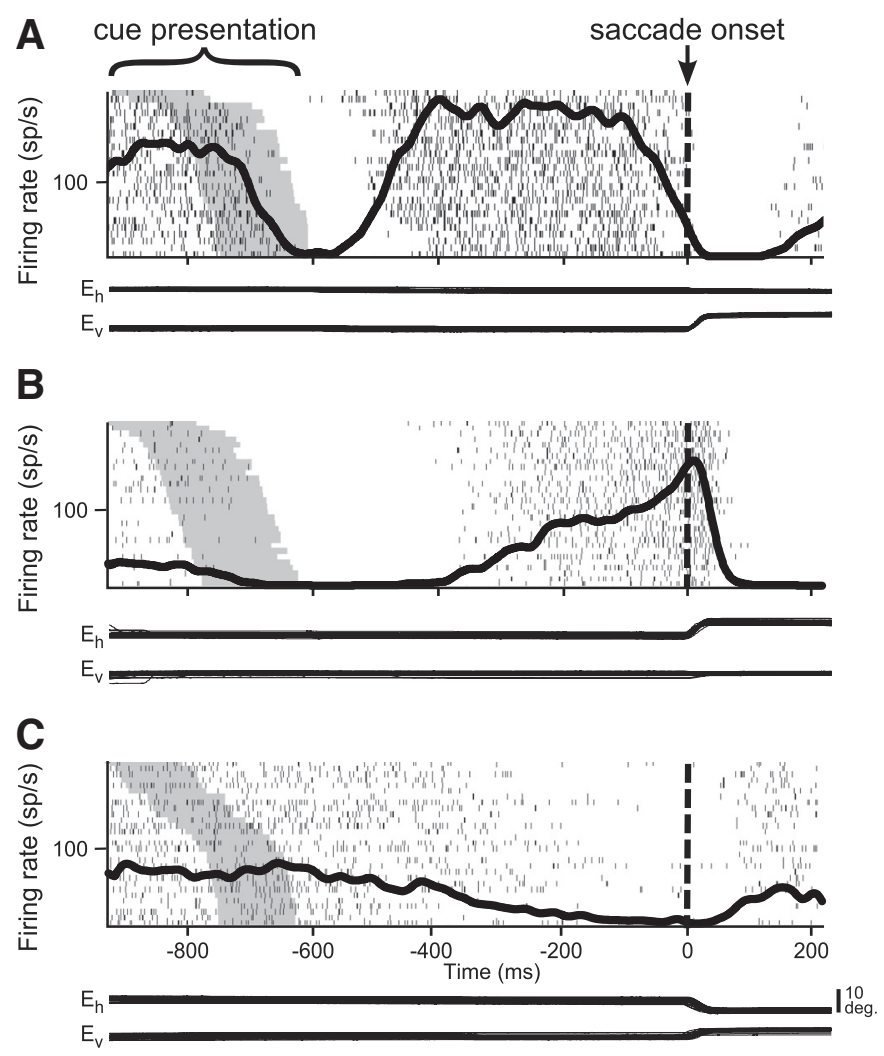

Fig. 3. Activity profiles of three example neurons recorded from caudal DN during the VS task, along with corresponding eye position traces. Superimposed are rasters showing the times of action potentials in individual trials and spike density functions (Gaussian kernels, 20-ms width) showing average firing rates. The data are aligned to saccade onset (time zero, vertical dashed line); gray areas indicate visual cue presentation times in each trial. $\mathrm{E}_{\mathrm{h}}$ and $\mathrm{E}_{\mathrm{v}}$ traces show, respectively, the horizontal and vertical components of eye position. Neuronal activity changed substantially after cue presentation and before saccade onset (i.e., during the delay period), while eye position remained steady. These three neurons illustrate typical sustained $(A)$, upward ramping $(B)$, and downward ramping $(C)$ delay activity patterns. sp/s, Spikes per second.

\section{General Characteristics of Delay Activity in the Caudal DN}

A neuron was considered to have delay activity if its firing rate during the delay period differed significantly from its baseline activity before the delay period (see METHODS). We attempted to test every neuron with the VS, DVG, and AS tasks, in that sequence of priority (the MG task was used on a subset of neurons as discussed later in this section). We found that $84 \%(66 / 79)$ of the caudal DN neurons that we recorded had significant delay activity for at least one task and direction (paired $t$-tests, $P<0.05$, Bonferroni corrected for multiple comparisons).

The VS and AS tasks were different with respect to the sensory cues that specified where the saccade should go, but for both tasks the saccade had to be self-timed. Of the 66 neurons with significant delay activity, 42 were tested on both tasks, and significant delay activity was present in both tasks for nearly all $(93 \%, 39 / 42)$ of these neurons. As represented in a typical example neuron (Fig. 4A), the time course of the delay activity (e.g., upward ramping) was always the same between tasks, although the relative strengths of the activity could differ. We quantified this strength difference for 33 neurons that had significant delay activity in one or both tasks, for directions that yielded at least eight trials in each 

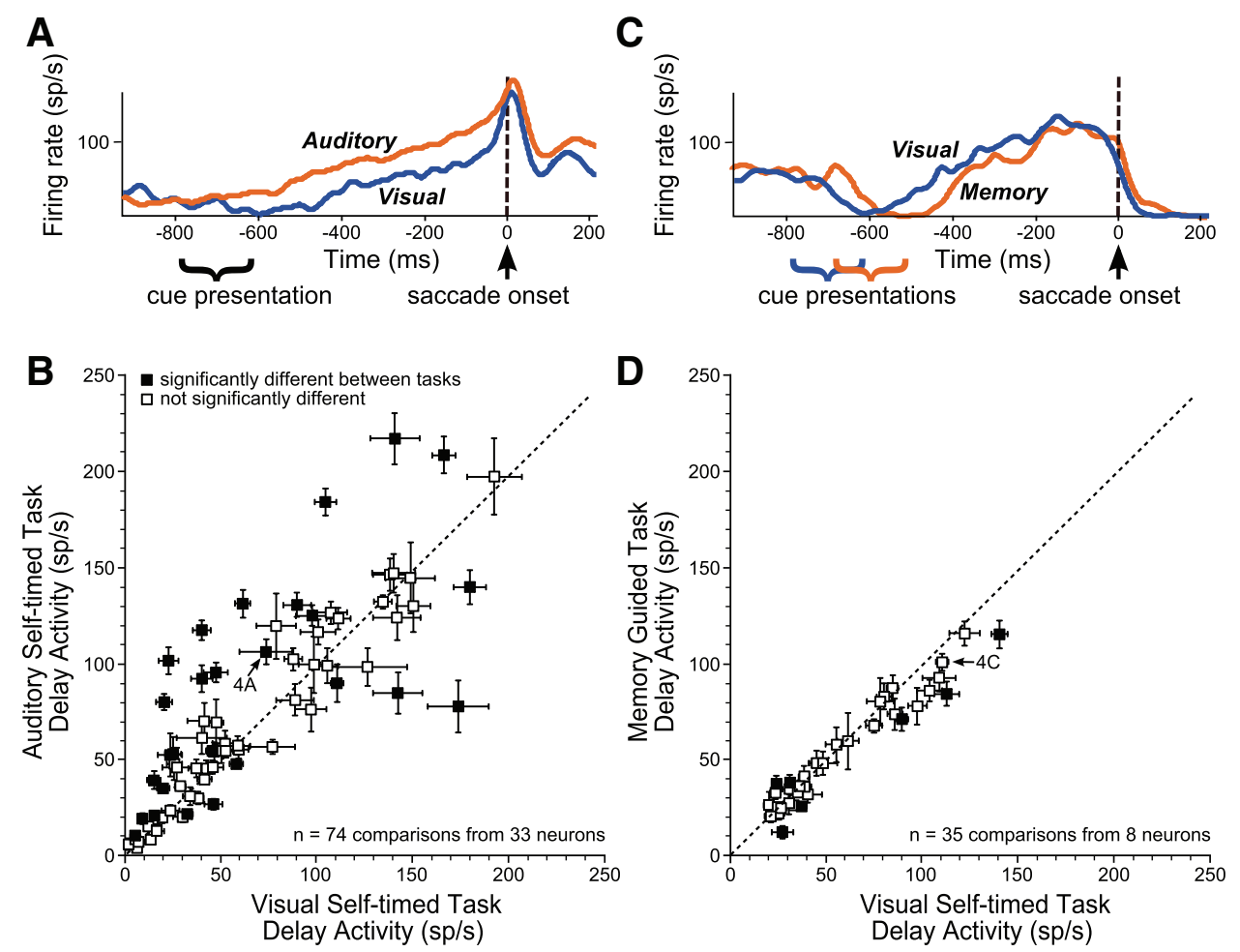

Fig. 4. Comparisons of VS task data with AS and MG task data. $A$ : example neuron tested on the VS and AS tasks. Average activity profiles evoked by the two tasks for the same direction of movement are shown using superimposed spike density functions aligned to saccade initiation. $B$ : delay activity for all neurons and directions tested on both the VS and AS task. A data point was included if delay activity was significant for the VS task, AS task, or both, and if there were at least 8 trials from both tasks. Symbols show mean and SE of the delay activity, as measured from -300 to $0 \mathrm{~ms}$ relative to saccade initiation in both tasks. Black squares show individual data for which delay activity was significantly different between the VS and AS tasks $(t$-test, $P<0.05)$, and white squares show data for which delay activity in the two tasks was not significantly different. Data point from $A$ is labeled " $4 \mathrm{~A}$." Dashed line is the unity line. $C$ : same as $A$, but showing data from an example neuron and direction tested on both the VS and MG tasks. $D$ : same as $B$, but comparing delay activity evoked by the VS and MG tasks; data point from $C$ is labeled "4C."

task; this yielded a total of 74 comparisons (Fig. $4 B$ ). For most of the comparisons $(64 \%, 47 / 74)$, the strength of the delay activity was not significantly different between the tasks ( $t$-test, $P>0.05$ ). Delay activity was greater when cued by sound for $27 \%(20 / 74)$ and greater when cued by vision for $15 \%(7 / 47 ; P<0.05)$ of the comparisons. At the population level, stronger delay activity in the auditory condition was significant (medians 58.0 and 46.7 spikes/s, respectively; Wilcoxon signed-rank test, $P=0.004$ ). The two tasks, therefore, elicited qualitatively similar patterns of delay activity in caudal DN neurons, except that the activity was generally larger for auditory targets than for visual targets.

The VS, DVG, and MG tasks were all similar in using visual cues, but they differed in that the VS task required self-timing of saccades, while the DVG and MG tasks did not. The DVG task was the simplest comparison condition in that it lacked not only the requirement to self-time, but also fixation point disappearance. The MG task does not require self-timing, but Tanaka (2007) demonstrated that monkeys may treat it as a self-timed task in which they predict when the fixation spot will disappear. Consistent with Tanaka's (2007) results, we found that delay activity profiles evoked during the VS and MG tasks in caudal DN were nearly identical. Figure $4 C$ shows data from a typical example neuron. Delay periods were shorter on average in the MG than in the VS task (see METHODS), so there was a slight temporal offset between the start of the delay activity. Nevertheless, the activity profiles were indistinguishable by the time of saccade initiation. We tested the MG task on eight neurons (4 from each monkey), using eight directions as in the VS task (Fig. 4D). Delay activity evoked by the MG task closely tracked that evoked by the VS task, with no significant difference between the two distributions (medians 41.6 and 40.0 spikes/s, respectively; Wilcoxon signed-rank test, $P=0.08$ ). Note, although we attribute the similar delay activity in MG and VS tasks to implicit self-timing in the MG task (Tanaka 2007), other explanations are possible. For example, both tasks might recruit common, general motor preparatory processes (addressed in the DISCUSSION). In any case, due to the time constraints of studying isolated neurons and the redundancy of the VS and MG task data, we discontinued use of the MG task. The rest of our report focuses on data from the VS, AS, and DVG tasks.

Many neurons displayed a transient decline in firing rate, or "pause", prior to the appearance of significant delay activity (e.g., Fig. 3, $A$ and $B$ ). Of the 66 neurons with significant delay period activity, 58\% (38/66) showed significant pauses (see METHODS); their mean delay period activity (for at least one task and direction) dropped more than $2 \mathrm{SD}$ below the mean baseline for at least $50 \mathrm{~ms}$ and then returned to within $2 \mathrm{SD}$ of baseline during the delay period. These pauses could be dramatic: for 36\% (24/66) of the neurons, the activity dropped more than 4 SD below mean baseline before returning to within $2 \mathrm{SD}$ of baseline. The average pause duration (activity continuously more than $2 \mathrm{SD}$ below mean baseline) was $155.0 \mathrm{~ms}$ (SD $71.1 \mathrm{~ms}$ ).

Our analyses of delay activity might be skewed by comparing it with baseline activity, because if the neurons encode preparatory processes, such as predictive timing of task events, baseline activity may itself be modulated (e.g., in anticipation of the start of the delay period). In a second analysis, therefore, we performed a bootstrap test of activity solely within the delay period (details in METHODS). The test identified significant modulations in delay activity, such as upward ramps, downward ramps, or more complex changes (e.g., bimodal, updown-up profiles). Performing this analysis on the 66 neurons considered above, it identified 44 of the neurons $(67 \%)$ as having significant temporally structured delay activity (z-test, $P<0.03)$. Note that the bootstrap test cannot identify neurons having delay activity that is temporally unmodulated (i.e., sustained), yet different from baseline, so the smaller propor- 
tion of neurons identified as having delay activity according to this test, relative to the proportion found with the baselinecomparison test, is to be expected.

\section{Trial-by-Trial Correlations Between Activity and Saccade Latency in the Caudal DN}

We found that caudal DN neurons with significant delay activity often exhibited distinct changes in firing rates that were correlated with saccadic latency on a trial-by-trial basis. "Saccadic latency" in our study is relative to the start of the delay period. Data collected during the VS task from three example neurons are shown in Fig. 5A. Two of the neurons abruptly stopped their delay activity in correlation with saccades, one just after saccade onset (Fig. 5A, top) and the other coincident with saccade onset (Fig. $5 A$, middle). A third neuron (Fig. 5A, bottom) suddenly increased its activity just after saccade onset. To quantitatively analyze these correlations for each neuron, we defined the time of the identifiable feature of activity on each trial (relative to the start of the delay period) as the "neuronal latency". To ensure objectivity of the analysis, we used an automated algorithm to extract measurable features (firing rate threshold crossings and inflections, see METHODS) and computed the neuronal latency of every neuron in every trial. We then calculated the Pearson's correlation between neuronal latency and saccade latency for all trials and every test condition (task and direction). All three of the neurons in Fig. 5A exhibited a highly significant correlation between neuronal and saccade latency for the conditions shown (results at top right of each graph).

For population analyses, we analyzed the data for each direction that had sufficient data for correlation tests (at least 8 trials) from each neuron separately, yielding 381 correlation tests for the VS task (66 neurons), 149 tests for the AS task (45 neurons), and 306 tests for the DVG task (59 neurons). The distributions of correlation coefficients (Fig. 5, B-D) had medians that were significantly positive (greater than zero) for data collected in all three tasks (VS: 0.24; AS: 0.16; DVG: 0.12; Wilcoxon signed-rank tests, all $P<0.001$ ), but the distributions differed between tasks (ANOVA on ranks, $P=$ 0.004). Neuronal-saccadic latency correlations were significantly stronger for the VS task than for both the AS and DVG tasks (Dunn's multiple-comparison tests, $P<0.05$ ), but correlations were not significantly different between the AS and DVG tasks $(P>0.05)$. Corroborating results were found by considering the data on a neuron-by-neuron basis, as well. Correlations were significant in at least one direction for $53 \%$ $(35 / 66)$ of neurons tested on the VS task, 33\% (15/45) of neurons tested on the AS task, and $27 \%$ (16/59) of neurons tested in the DVG task. These proportions differed signifi-
Fig. 5. Correlations between neuronal modulation and saccade initiation. $A$ : delay activity profiles elicited during the VS task for three neurons (top, middle, and bottom) aligned to the start of the delay period (time zero; target cue offset is depicted with thick vertical lines) and sorted by saccadic latency (saccade initiation is depicted, trial by trial, with grey fiducial marks). For each neuron, the data are from a single direction. Below each raster set is a gray-scale thermal plot of individual spike density functions for each trial, to aid in visualizing trial-by-trial firing rate patterns. For all three neurons, the timing of delay activity (as tracked using an automated algorithm that identified common features of the activity across trials) was tightly correlated with saccade initiation (Pearson's correlation coefficients and $P$ values are shown at top right of each data set). $B-D$ : the correlation coefficient $(r)$ for every direction tested in every neuron during the VS, AS, and DVG task, respectively. Individually significant correlations are depicted in black. The median $r$ for each distribution is shown. *Different from zero at $P<0.05$.

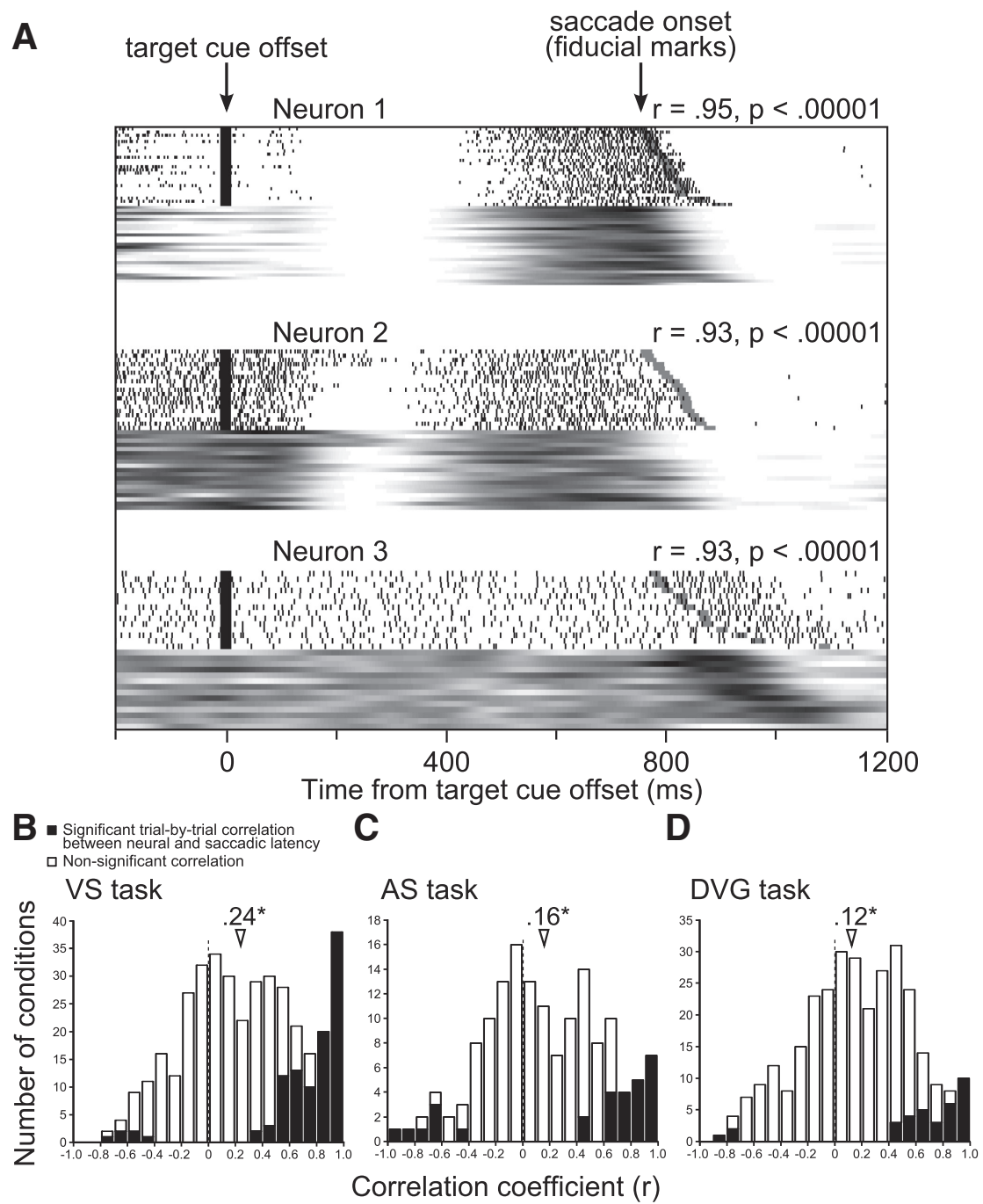


cantly between the tasks ( $\chi^{2}$ test, $\left.P=0.008\right)$, with more neurons showing correlations in the VS than in the DVG tasks (Fisher exact test, $P=0.004$; the other pairwise results were not significant: VS vs. AS, $P=0.052$, and AS vs. DVG, $P=$ 0.49). In a subset of 44 neurons for which we had sufficient data for correlation analyses from all three tasks, the results were essentially the same: correlations were significant for at least one direction in $48 \%$ (21/44) of the neurons for the VS task, in 32\% (14/44) of the neurons in the AS task, and in 23\% of the neurons in the DVG task (significant difference in proportions between tasks: $\chi^{2}$ test, $P=0.044$; significant pairwise comparison between VS and DVG tasks, $P=0.025$; nonsignificant pairwise comparisons between VS and AS tasks, $P=0.19$, and between AS and DVG, $P=0.47$ ).

\section{Directional Tuning of Caudal DN Delay Activity}

For many neurons, the strength of the delay activity clearly varied with direction (Fig. 6). We performed two analyses to quantify direction selectivity for the 66 neurons having significant delay activity. The purpose of the first analysis was to assess the incidence and timing of direction selectivity through a comparison of activity across all tested directions at successive points in the delay period. We limited this analysis to the VS task, for which eight directions were randomized by trial (in the AS task only 4 directions were randomized, and in the DVG task the direction was unspecified during the delay period). For each neuron, the delay activity for each direction was binned in six sequential epochs each $100 \mathrm{~ms}$ long, starting from target cue onset. Within each bin we performed an ANOVA on the activity across the directions $(P<0.05$ criterion corrected for 6 tests per neuron, i.e., $P<0.0083$ ). We found that direction selectivity was present during at least one bin for $65 \%$ (43/66) of neurons.

Direction selectivity was more common later in the delay period (Fig. 7A). Inspecting the data from individual neurons, it was clear that the selectivity settled over time into its stable, final tuning. Figure $7 B$ shows this effect for two neurons that had significant direction selectivity in all six bins. For each neuron, tuning in the first 100-ms bin of the delay period (black arrow) changed markedly in the next 100 -ms bin (dark gray arrow), rotating toward the final tuning of the last 100 -ms bin (light gray arrow). A total of nine neurons had significant direction selectivity in all six bins, and the tuning angle within each bin, relative to that in the first bin, is plotted in Fig. $7 C$ for them. Tuning angle changed significantly from the first $100-\mathrm{ms}$ period to the second, and from the second to the third, but not thereafter (paired $t$-tests, $P<0.01$ to correct for 5 tests between the 6 successive epochs).

We summarized these effects by calculating the average difference in angle from bin to bin for the nine neurons in Fig. $7 D$ and for the entire sample of neurons in Fig. $7 E$ (means and SEs; we used absolute values of the differences to disregard whether rotations were clockwise or counterclockwise). For the data from the entire sample, the tested numbers varied because we could only calculate the difference between se-

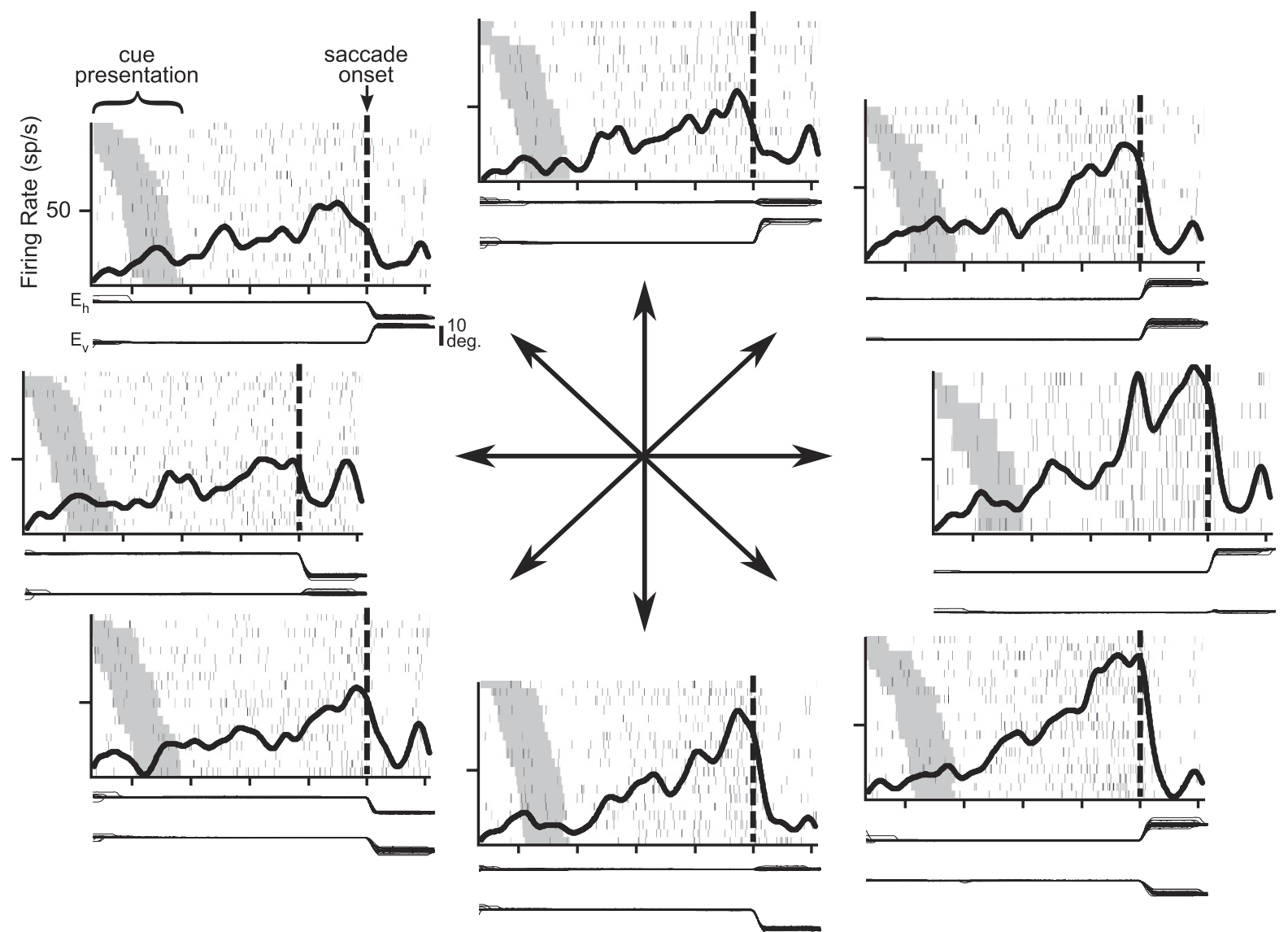

Fig. 6. Example neuron that had directionally selective delay activity. Data are from the VS task, sorted by target direction as depicted in central schematic. Eye position traces for each data set are shown as horizontal $\left(\mathrm{E}_{\mathrm{h}}\right)$ and vertical $\left(\mathrm{E}_{\mathrm{v}}\right)$ components, as in Fig. 3. Firing rate and eye position scales, shown for upper left data set, are the same for all data sets. Ticks on the $x$-axes are $200 \mathrm{~ms}$ apart. 

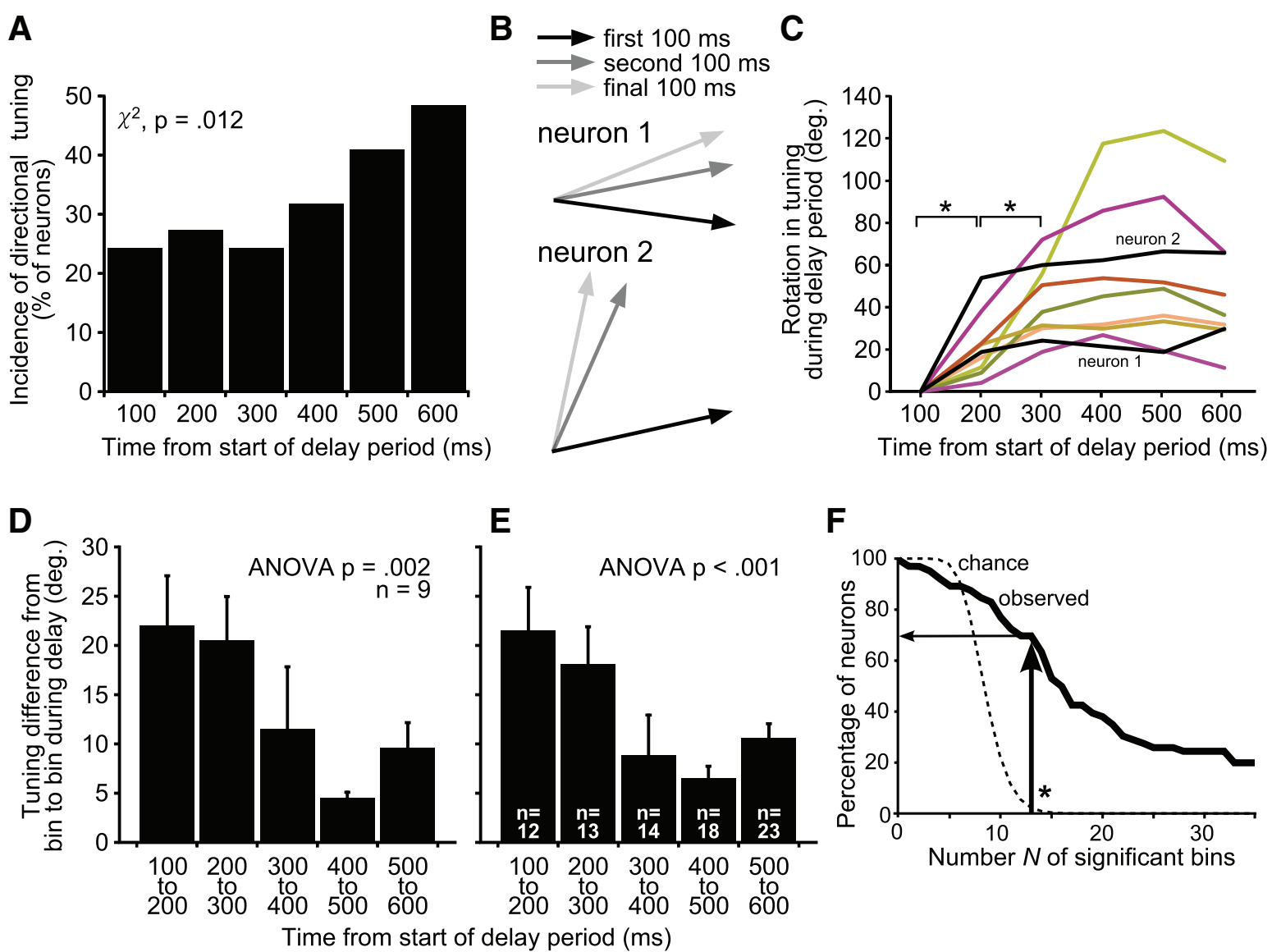

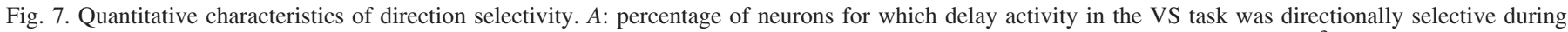

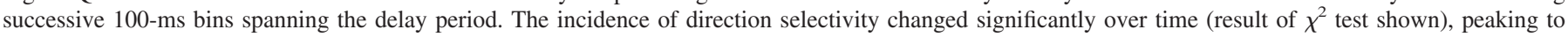

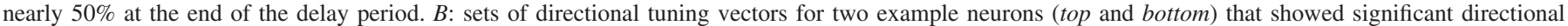

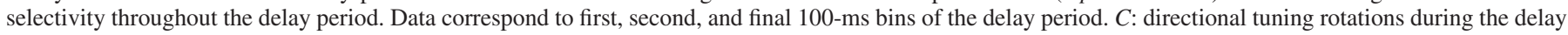

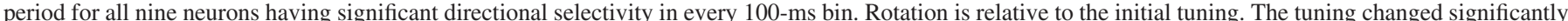

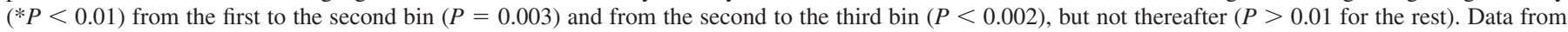

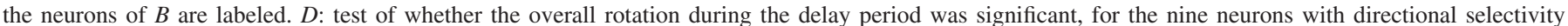

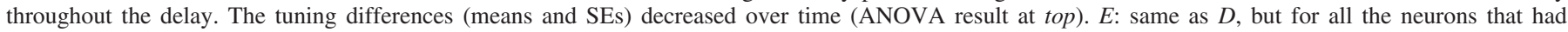

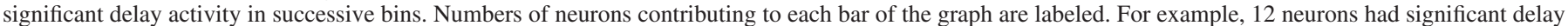

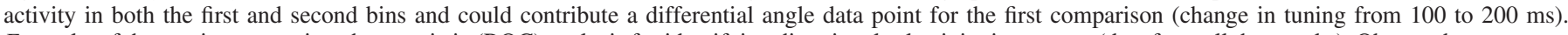

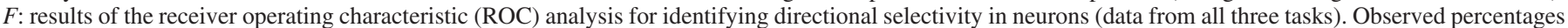

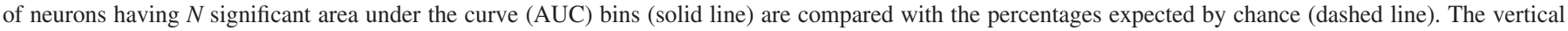

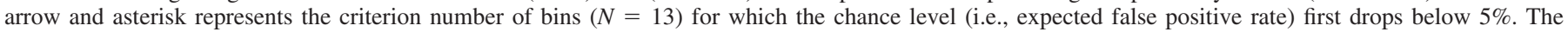
horizontal arrow shows that $70 \%$ of our neurons had 13 or more significant AUC bins and thus may be considered directionally selective at $P<0.05$.

quential bins if both had significant tuning, and most neurons (aside from the nine in Fig. 7, $C$ and $D$ ) had significant tuning in only a few bins. The numbers of neurons contributing to each bin's data in Fig. $7 E$ are shown explicitly. The plots of Fig. 7, $D$ and $E$, were nearly identical, demonstrating that the effect found for the subset of nine neurons was also present in the overall sample: tuning angles differed by $>20^{\circ}$ on average between the first and second 100-ms periods of the delay period, but stabilized to differ by $\sim 10^{\circ}$ or less between bins later in the delay period. The final tuning angles of the neurons could be oriented in any direction; we found no obvious contralateral, ipsilateral, or vertical biases.

In our second method for studying direction selectivity, we reduced our analysis from a simultaneous consideration of all directions (in the above tests) to a more focused analysis on the two directions that elicited the most distinctly different patterns of delay activity. For this we used an ROC bootstrap test (Green and Swets 1966; Horwitz and Newsome 2001; Phillips and Seg- raves 2010). As described in METHODS, for each neuron we found the two most distinct directions using a first-pass ROC analysis. Then we compared the entire time courses of delay activity for these two directions using a sliding ROC analysis in 5-ms increments and, for comparison, calculated the results that would be expected by chance. The results are shown in Fig. $7 F$. The dashed line shows the percentages of neurons expected to show $N$ bins of significant activity differences simply by chance. For example, all neurons were expected to have $N=5$ bins with significant ROC results just due to random fluctuations. As $N$ increases, the first time the chance level drops below $5 \%$ (i.e., $P<0.05$ false positive rate) is at $N=13$. Precisely only $2.16 \%$ of neurons would be expected to have 13 significant bins just by chance. In our sample of neurons, however, $70 \%$ (46/66) had 13 or more significant bins and, therefore, showed significant directional selectivity according to this analysis.

The ROC analysis of Fig. $7 F$ searched for direction selectivity in data from all of the tasks run on a neuron. We also ran 
the ROC analysis on data from the VS task alone (as done for the first, ANOVA-based analysis). The result was that 53\% (37/66) of the neurons were considered directionally selective. One implication of this result is that the VS task alone is sufficient for identifying direction selectivity for the great majority of neurons. Only 9 neurons (46 minus 37) had directional selectivity that would be missed by analyzing only VS task data. This supports the validity of the results found with the first, ANOVA-based analysis (which used only VS task data). Note also that there was reasonable correspondence between the final proportions of neurons found to have significant directional selectivity in the VS task for the two methods (53\% for ROC test vs. 65\% for the ANOVA-based test), despite the extremely different methodologies.

The ROC analysis yielded fine-scale temporal information as to when direction selectivity emerged during the delay period. It revealed that, on average, delay activity became significantly different between the best and worst tested directions $\sim 400 \mathrm{~ms}$ ( $393 \pm 229 \mathrm{~ms}$ ) before the start of the saccade. This is comparable to the rough time course we found with our ANOVA-based analysis, in which direction tuning was found to settle into its final state after the first $200 \mathrm{~ms}$ of the delay period $(400 \mathrm{~ms}$ before saccade onset is $\sim 300 \mathrm{~ms}$ into the 600 -ms delay period, depending on saccadic latency).

\section{Comparisons of Caudal DN Delay Activity in Tasks That Do} or Do Not Require Self-timing

In the VS task, a monkey was required to self-time; it had to wait for at least $600 \mathrm{~ms}$ and then, with no external cue to move, initiate its saccade. In the DVG task, there was no self-timing requirement. The monkey simply fixated for $600 \mathrm{~ms}$ and then made a saccade in response to visual target appearance. Fiftysix of the neurons with delay activity in the VS task were also tested with the DVG task, and we found that $89 \%$ (50/56) of those neurons exhibited significant activity during the delay period of that task. The delay activity in the two tasks, however, often had markedly different temporal profiles. An example neuron produced downward ramping activity in the VS task (Fig. 8A, top), but upward ramping activity in the DVG task (Fig. $8 A$, bottom). Because the differences were often time-varying as for the example neuron, they could be missed by averaging over an epoch. Hence we compared activity profiles between the VS and DVG task data over the 600-ms delay using a ROC bootstrap analysis (similar to that described in the previous section, except that here the $P<0.05$ criterion level corresponded to $N=14$ bins; Fig. $8 B)$. We found that $71 \%(40 / 56)$ of the neurons showed significantly different time courses of delay activity between the VS and DVG tasks for at least one direction (Fig. 8B). These task-related differences started on average $366 \mathrm{~ms}( \pm 240 \mathrm{~ms})$ before saccade onset. Most of the neurons, therefore, produced profiles of activity during imposed delays that differed when self-timing was (VS task) or was not (DVG task) required.

\section{Sensory Responses in the Caudal DN}

All of the above analyses focused on the delay activity, but we also searched for evidence of activity that might be tightly related to sensory events and saccades. Saccade-related activity is typically evaluated in delayed response tasks (e.g., Sommer and Wurtz 2000) by comparing firing rates just prior to saccade

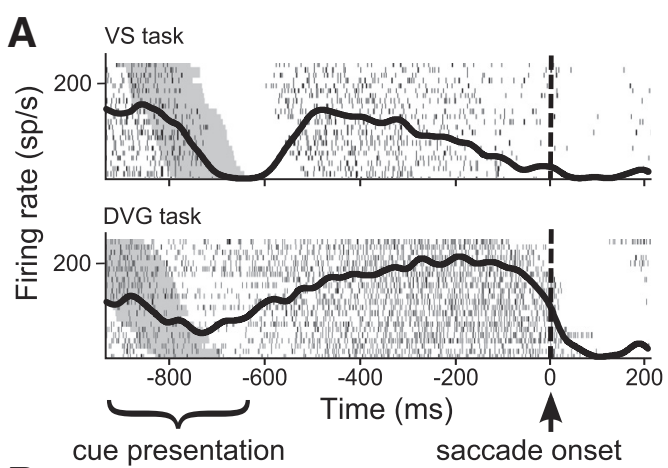

B

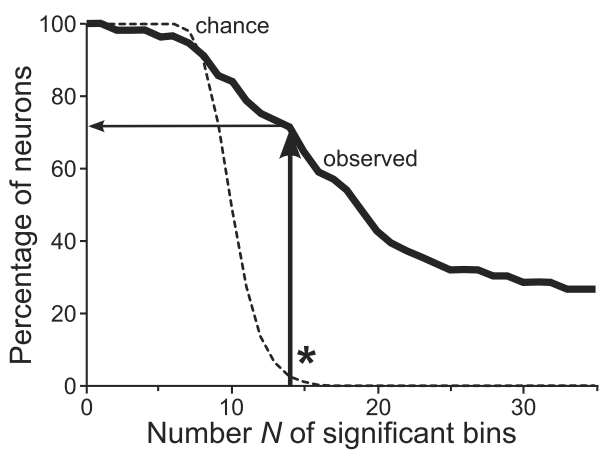

Fig. 8. Differences in temporal profiles of delay activity evoked by the VS and DVG tasks. Self-timing was required in the VS task, but was eliminated as thoroughly as possible in the DVG task. A: data from an example neuron are shown for the VS task (top) and the DVG task (bottom). The time course of the delay activity differed markedly in the two task contexts. $B$ : ROC analysis of VS-DVG task selectivity. Conventions are as for Fig. $7 F$, except that, in this analysis, the criterion number of bins that yielded $<0.05$ chance of false positive was $N=14$.

initiation with those during the delay period and a baseline period. This is appropriate for regions like FEF, in which neurons often show a sudden presaccadic burst after the delay, but it was inappropriate for our caudal DN neurons with their steady or ramping delay activity that continued up to or through the saccade. It was obvious by inspection that few if any of the caudal DN neurons showed discrete presaccadic bursts of activity comparable to those found in areas such as the FEF.

Phasic sensory-related responses, however, were clearly present in some neurons. We quantified sensory-related responses by comparing activity in an epoch 50-150 ms after a sensory cue (visual stimulus in VS task or auditory stimulus in AS task) with baseline activity in an epoch $100-0 \mathrm{~ms}$ before the cue (paired, one-tailed $t$-test, $P<0.01)$. The analysis was constrained to a subset of 45 neurons that had sufficient VS and AS trials ( $\geq 8$ for one or more directions) to permit valid analyses of responses to both visual and auditory cues. We found that $42 \%$ of the neurons (19/45) had a putative sensory response for one or more directions. For $21 \%$ of this subset (4/19 neurons), the sensory response was exclusively visual (VS trials only), for 53\% (10/19) the sensory response was exclusively auditory (AS trials only), and in $26 \%$ of the neurons $(5 / 19)$, sensory responses occurred for both tasks.

\section{Saccade-related Neurons Recorded More Rostrally in the DN of the Same Monkeys}

Previous, quantitative analyses of saccade-related neurons in the DN revealed activity patterns that were very different from 
what we found (Gardner and Fuchs 1975; Hepp et al. 1982; MacKay 1988). Such DN neurons, termed "short-lead bursters" (SLBs) by Hepp et al. (1982), fired a burst of action potentials that began shortly before saccade onset and lasted for the duration of the saccade (Gardner and Fuchs 1975; Hepp et al. 1982). They were distributed widely in the DN (MacKay 1988; Thach 1978; van Kan et al. 1993) and, therefore, should reside largely rostral to the caudal-pole neurons that we studied.

To directly compare our caudal DN neurons with previously reported SLBs, we searched for saccade-related neurons in penetrations of more rostral DN. We found nine saccaderelated neurons in a range $3-5 \mathrm{~mm}$ rostral to our caudal DN population. All nine matched the reported properties of SLB neurons. An example SLB is shown in Fig. 9, with data aligned on saccades made during the VS task (Fig. 9A) and saccades made outside of any task context (during intertrial intervals; Fig. 9B). The neuron was representative; all of the SLB neurons simply fired a burst of activity for any saccade. We collected full data files on four of the neurons. The presaccadic bursts of activity started $17.4 \pm 12.8 \mathrm{~ms}$ prior to saccade initiation, closely matching the lead times reported previously (Chubb and Fuchs 1982; Gardner and Fuchs 1975; Hepp et al. 1982; MacKay 1988). We did not exhaustively analyze the data because it was clear by inspection that the activity was the same as described in previous reports. The bursts lasted for the saccade duration, were omnidirectional (no direction tuning), were never preceded by delay activity, did not vary by task, and were present for saccades made outside of tasks (during intertrial periods). Albeit mostly qualitative, these findings demonstrate that our caudal DN neurons are not classical SLBs. The caudal DN neurons that we found seem to represent a markedly different, previously unreported population.
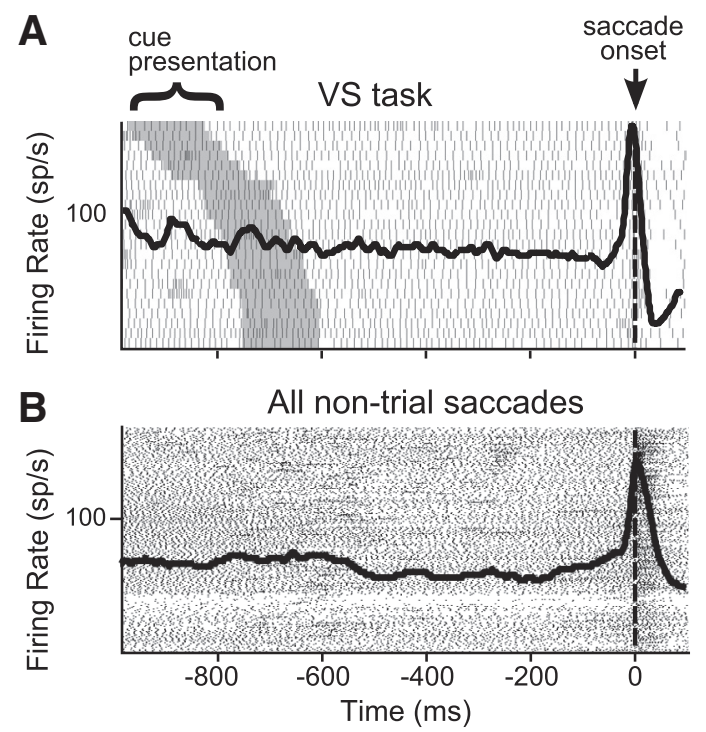

Fig. 9. Example of a short-lead burster (SLB) neuron recorded more rostral to the other neurons of our study. A: the neuron's activity during the VS task was dominated by a distinct presaccadic burst of activity. The neuron exhibited no delay activity. $B$ : activity for the same neuron was nearly identical for spontaneous saccades made during intertrial intervals. This example neuron was representative of our sample of nine SLB neurons. All of them lacked delay activity and fired in relation to all saccades, showing little or no modulation by task context.

\section{DISCUSSION}

In support of our hypothesis, we found that many caudal DN neurons have vigorous delay activity. The neurons 1 ) showed changes in firing rate that were temporally correlated with saccade initiation; 2) exhibited directional tuning with a continuous rotation of tuning angle during the delay; and 3) differentiated through their activity profiles between a task that required self-timing and one that did not. In general, the caudal DN seems to be a saccade-related domain where neurons convey prolonged signals related to the planning of when and where to move.

Historically, the cerebellum has been generally considered a "motor structure" that controls and adjusts movement parameters (Evarts and Thach 1969; Ito 2002; Strick et al. 2009). Many cerebellar areas conform to this classical view, such as the caudal fastigial nucleus with its well-documented control of saccade dynamics and endpoint (Fuchs et al. 2010; Goffart et al. 2003; Robinson and Fuchs 2001). The caudal DN, in contrast, seems more involved with long-lead preparation of when and where to move. Simply put, while recording from caudal DN neurons, we had the strong impression of finding a piece of prefrontal or parietal cortex in the depths of the cerebellum. Delay activity was originally discovered in prefrontal cortex (and the thalamic regions associated with it; Fuster and Alexander 1971), and since then it has been studied in many areas, including primary motor cortex (Tanji and Evarts 1976), premotor cortex (Weinrich et al. 1984), parietal cortex (Chafee and Goldman-Rakic 1998), inferotemporal cortex (Fuster and Jervey 1982), and the SC (Wurtz et al. 2001). Given that the DN connects with all of these areas, except inferotemporal cortex, and that the caudal DN specifically contributes to circuits involving prefrontal and parietal cortex and projects to the SC (May et al. 1990; Prevosto et al. 2007, 2010; Strick et al. 2009), it is not entirely surprising that we found delay activity in our neurons. It seemed extraordinary, however, that delay activity was by far the dominant type of activity present.

No prior, quantitative reports of oculomotor activity in the DN used delayed response tasks, reported the firing rate profiles that we found, or concentrated on the caudal pole (Chubb and Fuchs 1982; Gardner and Fuchs 1975; Hepp et al. 1982; MacKay 1988). More broadly, our study seems to be the first to document delay activity in the DN, if not in the entire cerebellum. The most relevant prior work was by Strick (1983) and Chapman et al. (1986). Both studies examined arm movement activity in DN. Strick (1983) reported that some DN neurons exhibit phasic, movement-related activity that is influenced by instructions. However, they did not report the rostrocaudal locations of their "set-related" DN neurons. Chapman et al. (1986) studied neurons in the caudal DN, seemingly close to the zone that we studied. Their tasks did not impose delays, but the activity profiles that they observed resembled time-compressed versions of the activity patterns we found: high baseline firing rates, often a pause just after a sensory cue, and often quick but smooth ramps up and/or down during the movement. Their movement-related activity often had peaks or troughs that correlated with movement initiation (their Figs. 6-10) and often had directional selectivity (their Fig. 8). They encountered eye movement-related neurons, notably in the caudal one-third of the DN, but did not study them. 
Delay activity is sometimes interpreted as representing the duration of temporal intervals (elapsed or anticipated), as contributing to the planning of timed movements, or as playing a role in temporal processing more generally (Durstewitz 2004; Janssen and Shadlen 2005; Lebedev et al. 2008; Lee and Assad 2003; Sakurai et al. 2004; Tanaka 2007). A related hypothesis is that the activity implements a temporally precise inhibitory control over movement (Ballanger 2009; Lo et al. 2009; Narayanan and Laubach 2009). Our data cannot distinguish between these hypotheses. If caudal DN delay activity contributes to the timing of movement, it could be encoding the elapsed time, suppressing premature movements, or both. For example, the fluctuations of delay activity found in many of our neurons, typified by abrupt pauses followed by recovery, could be a sign that braking and preparatory functions are occurring sequentially. Primate DN neurons are functionally capable of contributing to both excitatory and inhibitory functions. Electrical stimulation in DN causes orthodromic responses in cerebral cortical neurons that are mostly excitatory, but sometimes suppressive (e.g., Holdefer et al. 2000), and the targeted cortical neurons could be excitatory pyramidals or inhibitory interneurons. Other routes of excitation and suppression include polysynaptic connections from the DN (including its caudal pole) to nuclei of the basal ganglia (Bostan and Strick 2010; Hoshi et al. 2005).

The rare sensory responses and lack of discrete saccaderelated bursts in caudal DN suggest that the delay activity of its neurons has little to do with sensory-related preparatory functions (e.g., attention or memory) or the direct generation of saccades. A more parsimonious explanation, taking together all of our analyses, was that it contributes to an explicit timing signal and/or temporally modulated inhibitory control. We do not mean to imply, however, that timing is the only possible role of caudal DN neurons. The delay activity we found is clearly a fusion of multiple signals, representing spatial aspects of movement (e.g., direction) as well as temporal aspects (e.g., time of onset). At the start of the delay period, directional tuning was relatively uncommon, and, when present, it poorly matched the final tuning of the neuron. But the tuning signal strengthened and rotated during the first half of the delay period to settle into a final tuning angle by the second half. We do not understand the reasons for this rotation, but it is reasonable to speculate that the caudal DN-associated network develops an initial "guess" at the proper direction that must be refined over time. This rotation in direction selectivity is another commonality between caudal DN and frontal cortex; similar, temporally changing rotations of directional tuning have been found in single neurons of premotor and primary motor cortex (Johnson et al. 1999; Mason et al. 1998). We did not test other spatial attributes, such as saccadic amplitude, but it seems plausible that signals related to them may be embedded in the delay activity as well.

In our most direct test of the hypothesis that caudal DN activity carries some representation of self-timing, we compared delay activity produced in the VS and DVG tasks. Self-timing was required by the former, but minimized as much as possible in the latter. Our ROC comparisons of data from the two tasks suggest that the self-timing requirement influences the delay activity of most caudal DN neurons, and that this task-dependent modulation emerges hundreds of milliseconds before saccade initiation. It should be noted that our
ROC analyses were conservative in the sense of being strictly tied to well-defined statistical significance, as determined by explicit calculation of expected false-positive curves. Note also that our conclusion that activity profiles differed between the VS and DVG task was not a trivial result of delay activity being absent in the latter task; delay activity appeared in both tasks, but had differing temporal profiles. A naive prediction would be that, if the delay activity were related to self-timing, it should be stronger in the VS task than in the DVG task. But this is not the only way that the signal could be carried, and it was not the code used by the caudal DN neurons. We do not know the type of activity difference that matters to downstream neurons or networks for the purpose of reading out a selftiming signal, and our ROC analyses made no a priori assumptions about this; the analysis simply established, objectively, that the activity patterns were statistically different. For comparison, activity patterns in the VS and MG tasks were nearly identical, possibly due to similar preparatory processes. We expected that motor preparation in the MG task would be limited to planning where to move, but monkeys may have planned when to move as well, since fixation point disappearance was predictable (Tanaka 2007). These issues led us to abandon the MG task.

The two self-timing tasks, the VS and AS tasks, differed only in the sensory nature of the cue that specified the eventual vector of saccade. Nearly all neurons that produced delay activity in the VS task also produced delay activity in the AS task. The main difference was that the latter activity was stronger; follow-up studies should pursue the reason for this auditory enhancement. We propose that the delay activity common to both tasks represented the task requirement common to both tasks, self-timing. Both tasks required working memory, as well, but it seems doubtful that this was the function of the delay activity. Our caudal DN neurons were relatively insensitive to sensory input, showing only infrequent responses to auditory or visual stimuli. Hence they do not seem well-suited for producing mnemonic traces of such stimuli. Moreover, while neuropsychological evidence has provided ample evidence that the lateral cerebellum contributes to selftiming, there is little indication that it plays a role in spatial working memory (reviewed by Bellebaum and Daum 2007; Lagarde et al. 2009).

We targeted caudal DN for recordings because of its known connections with association areas of cerebral cortex via relays in the thalamus. Consistent with this presumed connectivity, caudal DN neurons displayed activity that was strikingly similar to that found by Tanaka (2007), using similar tasks, in VL thalamus and particularly in its paralaminar part that includes the region known as "area X". Tanaka (2007) favored the hypothesis that ascending ramps of activity in this thalamic region during self-timing saccade tasks were due to basal ganglia input. This inclination was based on anatomical studies (Lynch et al. 1994) and on similarities between lesion effects in the basal ganglia (e.g., Coslett et al. 2010; Meck 2006) and the paralaminar VL/area X region (Tanaka 2006). The most notable effect of VL lesions (Tanaka 2006) is a dramatic deficit in a self-timed task: saccade initiation is delayed markedly. However, as Tanaka (2007) acknowledged, the thalamic region he studied receives input not only from the basal ganglia, but also from the cerebellum-from the caudal DN in particular (Lynch et al. 1994; Prevosto et al. 2010). In fact, the majority of 
afferents seem to be from the cerebellum (Middleton and Strick 2000), and lesions and inactivation of the cerebellum cause a variety of deficits in the timing of movements (e.g., impaired rhythmicity, interval discrimination, and timing of conditioned responses; reviewed by Mauk and Buonomano 2004). As such, we posit that the self-timing task ramping activity recorded in VL may in part originate in the basal ganglia, but it is at least as likely that much of this activity is cerebellar in origin. Likewise, the behavioral deficits following VL lesions may be related to the basal ganglia, but our current results and previous anatomy allow us to make an equally strong argument in favor of the cerebellum. An important next step will be to use antidromic activation methods to test whether the caudal DN neurons that we described do, in fact, project to VL thalamus.

\section{ACKNOWLEDGMENTS}

We thank T. Kevin Hitchens for MRI scans, Dr. Vincent Prevosto for helpful comments on an earlier draft of the manuscript, and Dr. Peter L. Strick and Michele O'Malley for invaluable assistance with histology.

\section{GRANTS}

Funded by NIH EY017592 to MAS and training grant NS007391 to RCA (PI: MJ Zigmond). MRI scans made possible by P41 EB001977 (PI: C Ho).

\section{DISCLOSURES}

No conflicts of interest, financial or otherwise, are declared by the author(s).

\section{AUTHOR CONTRIBUTIONS}

Author contributions: R.C.A. and M.A.S. conception and design of research; R.C.A. performed experiments; R.C.A. and M.A.S. analyzed data; R.C.A. and M.A.S. interpreted results of experiments; R.C.A. and M.A.S. prepared figures; R.C.A. and M.A.S. drafted manuscript; R.C.A. and M.A.S. edited and revised manuscript; R.C.A. and M.A.S. approved final version of manuscript.

\section{REFERENCES}

Alexander GE, DeLong MR, Strick PL. Parallel organization of functionally segregated circuits linking basal ganglia and cortex. Aпnи Rev Neurosci 9: 357-381, 1986.

Allen G, McColl R, Barnard H, Ringe WK, Fleckenstein J, Cullum CM. Magnetic resonance imaging of cerebellar-prefrontal and cerebellar-parietal functional connectivity. Neuroimage 28: 39-48, 2005.

Ashmore RC, Renk JA, Schmidt MF. Bottom-up activation of the vocal motor forebrain by the respiratory brainstem. J Neurosci 28: 2613-2623, 2008.

Ballanger B. Top-down control of saccades as part of a generalized model of proactive inhibitory control. J Neurophysiol 102: 2578-2580, 2009.

Bellebaum C, Daum I. Cerebellar involvement in executive control. Cerebellum 6: 184-192, 2007.

Bostan AC, Strick PL. The cerebellum and basal ganglia are interconnected. Neuropsychol Rev 20: 261-270, 2010.

Bottjer SW, Altenau B. Parallel pathways for vocal learning in basal ganglia of songbirds. Nat Neurosci 13: 153-155, 2010.

Bruce CJ, Goldberg ME. Primate frontal eye fields. I. Single neurons discharging before saccades. J Neurophysiol 53: 603-635, 1985.

Carello CD, Krauzlis RJ. Manipulating intent: evidence for a causal role of the superior colliculus in target selection. Neuron 43: 575-583, 2004.

Chafee MV, Goldman-Rakic PS. Matching patterns of activity in primate prefrontal area 8a and parietal area 7ip neurons during a spatial working memory task. J Neurophysiol 79: 2919-2940, 1998.

Chapman CE, Spidalieri G, Lamarre Y. Activity of dentate neurons during arm movements triggered by visual, auditory, and somesthetic stimuli in the monkey. J Neurophysiol 55: 203-226, 1986.
Chubb MC, Fuchs AF. Contribution of y group of vestibular nuclei and dentate nucleus of cerebellum to generation of vertical smooth eye movements. J Neurophysiol 48: 75-99, 1982.

Colby CL, Duhamel JR, Goldberg ME. Visual, presaccadic, and cognitive activation of single neurons in monkey lateral intraparietal area. $J$ Neurophysiol 76: 2841-2852, 1996.

Coslett HB, Wiener M, Chatterjee A. Dissociable neural systems for timing: evidence from subjects with basal ganglia lesions. PLos One 5: e10324, 2010.

Crapse TB, Sommer MA. Frontal eye field neurons with spatial representations predicted by their subcortical input. J Neurosci 29: 5308-5318, 2009.

Dias EC, Segraves MA. Muscimol-induced inactivation of monkey frontal eye field: effects on visually and memory-guided saccades. J Neurophysiol 81: 2191-2214, 1999.

Durstewitz D. Neural representation of interval time. Neuroreport 15: 745749, 2004.

Evarts EV, Thach WT. Motor mechanisms of the CNS: cerebrocerebellar interrelations. Аппи Rev Physiol 31: 451-498, 1969.

Farries MA. The avian song system in comparative perspective. Ann N Y Acad Sci 1016: 61-76, 2004.

Fuchs AF, Brettler S, Ling L. Head-free gaze shifts provide further insights into the role of the medial cerebellum in the control of primate saccadic eye movements. J Neurophysiol 103: 2158-2173, 2010.

Fuster JM, Alexander GE. Neuron activity related to short-term memory. Science 173: 652-654, 1971.

Fuster JM, Jervey JP. Neuronal firing in the inferotemporal cortex of the monkey in a visual memory task. J Neurosci 2: 361-375, 1982.

Gardner EP, Fuchs AF. Single-unit responses to natural vestibular stimuli and eye movements in deep cerebellar nuclei of the alert rhesus monkey. $J$ Neurophysiol 38: 627-649, 1975.

Goffart L, Chen LL, Sparks DL. Saccade dysmetria during functional perturbation of the caudal fastigial nucleus in the monkey. Ann N Y Acad Sci 1004: 220-228, 2003.

Green DM, Swets JA. Signal Detection Theory and Psychophysics. New York: Wiley, 1966.

Habas C. Functional imaging of the deep cerebellar nuclei: a review. Cerebellum 9: 22-28, 2010.

Habas C, Kamdar N, Nguyen D, Prater K, Beckmann CF, Menon V, Greicius MD. Distinct cerebellar contributions to intrinsic connectivity networks. J Neurosci 29: 8586-8594, 2009.

Helminski JO, Segraves MA. Macaque frontal eye field input to saccaderelated neurons in the superior colliculus. J Neurophysiol 90: 1046-1062, 2003.

Hepp K, Henn V, Jaeger J. Eye movement related neurons in the cerebellar nuclei of the alert monkey. Exp Brain Res 45: 253-264, 1982.

Hikosaka O, Wurtz RH. Visual and oculomotor functions of monkey substantia nigra pars reticulata. III. Memory-contingent visual and saccade responses. J Neurophysiol 49: 1268-1284, 1983.

Holdefer RN, Miller LE, Chen LL, Houk JC. Functional connectivity between cerebellum and primary motor cortex in the awake monkey. $J$ Neurophysiol 84: 585-590, 2000.

Hoshi E, Tremblay L, Féger J, Carras PL, Strick PL. The cerebellum communicates with the basal ganglia. Nat Neurosci 8: 1491-1493, 2005.

Horwitz DH, Newsome WT. Target selection for saccadic eye movements: prelude activity in the superior colliculus during a direction-discrimination task. J Neurophysiol 86: 2543-2558, 2001.

Huerta MF, Krubitzer LA, Kaas JH. Frontal eye field as defined by intracortical microstimulation in squirrel monkeys, owl monkeys, and macaque monkeys. I. Subcortical connections. J Comp Neurol 253: 415-439, 1986.

Ito M. Historical review of the significance of the cerebellum and the role of Purkinje cells in motor learning. Ann N Y Acad Sci 978: 273-288, 2002.

Ivry RB. The representation of temporal information in perception and motor control. Curr Opin Neurobiol 6: 851-857, 1996.

Ivry RB, Keele SW. Timing functions of the cerebellum. J Cogn Neurosci 1: $136-152,1989$.

Ivry RB, Keele SW, Diener HC. Dissociation of the lateral and medial cerebellum in movement timing and movement execution. Exp Brain Res 73: 167-180, 1988.

Janssen P, Shadlen MN. A representation of the hazard rate of elapsed time in macaque area LIP. Nat Neurosci 8: 234-241, 2005.

Johnson MT, Coltz JD, Hagen MC, Ebner TJ. Visuomotor processing as reflected in the directional discharge of premotor and primary motor cortex neurons. J Neurophysiol 81: 875-894, 1999. 
Kelly RM, Strick PL. Cerebellar loops with motor cortex and prefrontal cortex of a nonhuman primate. J Neurosci 23: 8432-8444, 2003.

Kim SG, Uğurbil K, Strick PL. Activation of a cerebellar output nucleus during cognitive processing. Science 265: 949-951, 1994.

Krienen FM, Buckner RL. Segregated fronto-cerebellar circuits revealed by intrinsic functional connectivity. Cereb Cortex 19: 2485-2497, 2009.

Küper M, Dimitrova A, Thürling M, Maderwald S, Roths J, Elles HG, Gizewski ER, Ladd ME, Diedrichsen J, Timmann D. Evidence for a motor and a non-motor domain in the human dentate nucleus-an fMRI study. Neuroimage 54: 2612-2622, 2011.

Lagarde J, Hantkie O, Hajjioui A, Yelnik A. Neuropsychological disorders induced by cerebellar damage. Ann Phys Rehabil Med 52: 360-370, 2009.

Lebedev MA, O'Doherty JE, Nicolelis MA. Decoding of temporal intervals from cortical ensemble activity. J Neurophysiol 99: 166-186, 2008.

Lee IH, Assad JA. Putaminal activity for simple reactions or self-timed movements. J Neurophysiol 89: 2528-2537, 2003.

Leichnetz GR. Inferior frontal eye field projections to the pursuit-related dorsolateral pontine nucleus and middle temporal area (MT) in the monkey. Vis Neurosci 3: 171-180, 1989.

Leon MI, Shadlen MN. Representation of time by neurons in the posterior parietal cortex of the macaque. Neuron 38: 317-327, 2003.

Li JX, Lisberger SG. Learned timing of motor behavior in the smooth eye movement region of the frontal eye fields. Neuron 69: 159-169, 2011.

Lo CC, Boucher L, Pare M, Schall JD, Wang XJ. Proactive inhibitory control and attractor dynamics in countermanding action: a spiking neural circuit model. J Neurosci 29: 9059-9071, 2009.

Lynch JC, Hoover JE, Strick PL. Input to the primate frontal eye field from the substantia nigra, superior colliculus, and dentate nucleus demonstrated by transneuronal transport. Exp Brain Res 100: 181-186, 1994.

MacKay WA. Cerebellar nuclear activity in relation to simple movements. Exp Brain Res 71: 47-58, 1988.

MacPherson JM, Aldridge JW. A quantitative method of computer analysis of spike train data collected from behaving animals. Brain Res 175: 183-187, 1979.

Mason CR, Johnson MT, Fu QG, Gomez JE, Ebner TJ. Temporal profile of the directional tuning of the discharge of dorsal premotor cortical cells. Neuroreport 9: 989-995, 1998 .

Mauk MD, Buonomano DV. The neural basis of temporal processing. Аnпu Rev Neurosci 27: 307-340, 2004

May PJ, Hartwich-Young R, Nelson J, Sparks DL, Porter JD. Cerebellotectal pathways in the macaque: implications for collicular generation of saccades. Neuroscience 36: 305-324, 1990.

Mays LE, Sparks DL. The localization of saccade targets using a combination of retinal and eye position information. In: Progress in Oculomotor Research, edited by Fuchs A and Becker W. New York: Elsevier, 1981, p. $39-47$.

McHaffie JG, Stanford TR, Stein BE, Coizet V, Redgrave P. Subcortical loops through the basal ganglia. Trends Neurosci 28: 401-407, 2005.

Meck WH. Neuroanatomical localization of an internal clock: a functional link between mesolimbic, nigrostriatal, and mesocortical dopaminergic systems. Brain Res 1109: 93-107, 2006.

Middleton FA, Strick PL. Anatomical evidence for cerebellar and basal ganglia involvement in higher cognitive function. Science 266: 458-461, 1994.

Middleton FA, Strick PL. Basal ganglia and cerebellar loops: motor and cognitive circuits. Brain Res Rev 31: 236-250, 2000.

Mikula S, Stone JM, Jones EG. BrainMaps.org - Interactive high-resolution digital brain atlases and virtual microscopy. Brains Minds Media 3: bmm1426, 2008.

Munoz DP, Wurtz RH. Saccade-related activity in monkey superior colliculus. I. Characteristics of burst and buildup cells. J Neurophysiol 73: 23132333, 1995.

Narayanan NS, Laubach M. Delay activity in rodent frontal cortex during a simple reaction time task. J Neurophysiol 101: 2859-2871, 2009.

Nummela SU, Krauzlis RJ. Inactivation of primate superior colliculus biases target choice for smooth pursuit, saccades, and button press responses. $J$ Neurophysiol 104: 1538-1548, 2010.

Onoe H, Komori M, Onoe K, Takechi H, Tsukada H, Watanabe Y. Cortical networks recruited for time perception: a monkey positron emission tomography (PET) study. Neuroimage 13: 37-45, 2001.

Parent A, Hazrati LN. Functional anatomy of the basal ganglia. I. The corticobasal ganglia-thalamo-cortical loop. Brain Res Rev 20: 91-127, 1995.

Perkel DJ. Origin of the anterior forebrain pathway. Ann N Y Acad Sci 1016: 736-748, 2004.
Phillips AN, Segraves MA. Predictive activity in macaque frontal eye field neurons during natural scene searching. J Neurophysiol 103: 1238-1252, 2010

Prevosto V, Graf W, Ugolini G. Cerebellar inputs to intraparietal cortex areas LIP and MIP: functional frameworks for adaptive control of eye movements, reaching, and arm/eye/head movement coordination. Cereb Cortex 20: 214-228, 2010.

Prevosto V, Ugolini G, Graf W. Cerebellar Control of Eye Movements: Polysynaptic Inputs From Fastigial, Interpositus Posterior and Dentate Nuclei to Lateral Rectus Motoneurons in Primates, Revealed by Retrograde Transneuronal Transfer of Rabies Virus (Abstract) (Online). Program No. 718.10. 2007 Neuroscience Meeting Planner. San Diego, CA: Society for Neuroscience, 2007. http:// www.abstractsonline.com/viewer/viewAbstract.asp?CKey $=\{822 \mathrm{~B} 7821-96 \mathrm{FF}-$ 4868-BCF3-1A43504B05A0 $\} \&$ MKey $=\{$ FF8B70E5-B7F9-4D07-A58A$\mathrm{C} 1068 \mathrm{FDE} 9 \mathrm{D} 25\} \& \mathrm{AKey}=\{3 \mathrm{~A} 7 \mathrm{DC} 0 \mathrm{~B} 9-\mathrm{D} 787-44 \mathrm{AA}-\mathrm{BD} 08-\mathrm{FA} 7 \mathrm{BB} 2 \mathrm{FE} 9004\}-$ $\&$ SKey $=\{$ E5F20097-7E6A-49FA-975F-91930ED09098 $\}$.

Robinson FR, Fuchs AF. The role of the cerebellum in voluntary eye movements. Annu Rev Neurosci 24: 981-1004, 2001.

Sakurai Y, Takahashi S, Inoue M. Stimulus duration in working memory is represented by neuronal activity in the monkey prefrontal cortex. Eur J Neurosci 20: 1069-1080, 2004

Salmi J, Pallesen KJ, Neuvonen T, Brattico E, Korvenoja A, Salonen O, Carlson S. Cognitive and motor loops of the human cerebro-cerebellar system. J Cogn Neurosci 22: 2663-2676, 2010.

Sasaki K, Jinnai K, Gemba H, Hashimoto S, Mizuno N. Projection of the cerebellar dentate nucleus onto the frontal association cortex in monkeys. Exp Brain Res 37: 193-198, 1979.

Sasaki K, Kawaguchi S, Oka H, Sakai M, Mizuno N. Electrophysiological studies on the cerebellocerebral projections in monkeys. Exp Brain Res 24: 495-507, 1976.

Sasaki K, Oka H, Kawaguchi S, Jinnai K, Yasuda T. Mossy fibre and climbing fibre responses produced in the cerebeller cortex by stimulation of the cerebral cortex in monkeys. Exp Brain Res 29: 419-428, 1977.

Schall JD, Hanes DP. Neural mechanisms of selection and control of visually guided eye movements. Neural Netw 11: 1241-1251, 1998.

Schall JD, Thompson KG. Neural selection and control of visually guided eye movements. Annu Rev Neurosci 22: 241-259, 1999.

Schmahmann JD. The Cerebellum and Cognition. New York: Academic, 1997.

Schmahmann JD, Pandya DN. Anatomic organization of the basilar pontine projections from prefrontal cortices in rhesus monkey. J Neurosci 17: 438-458, 1997.

Segraves MA, Goldberg ME. Functional properties of corticotectal neurons in the monkey's frontal eye field. J Neurophysiol 58: 1387-1419, 1987.

Sommer MA, Tehovnik EJ. Reversible inactivation of macaque frontal eye field. Exp Brain Res 116: 229-249, 1997.

Sommer MA, Wurtz RH. Composition and topographic organization of signals sent from the frontal eye field to the superior colliculus. J Neurophysiol 83: 1979-2001, 2000.

Sommer MA, Wurtz RH. Frontal eye field sends delay activity related to movement, memory, and vision to the superior colliculus. J Neurophysiol 85: 1673-1685, 2001.

Sommer MA, Wurtz RH. What the brain stem tells the frontal cortex. I. Oculomotor signals sent from superior colliculus to frontal eye field via mediodorsal thalamus. J Neurophysiol 91: 1381-1402, 2004.

Spencer RM, Zelaznik HN, Diedrichsen J, Ivry RB. Disrupted timing of discontinuous but not continuous movements by cerebellar lesions. Science 300: 1437-1439, 2003.

Stein JF. Role of the cerebellum in the visual guidance of movement. Nature 323: 217-221, 1986.

Strick PL. The influence of motor preparation on the response of cerebellar neurons to limb displacements. J Neurosci 3: 2007-2020, 1983.

Strick PL, Dum RP, Fiez JA. Cerebellum and nonmotor function. Annu Rev Neurosci 32: 413-434, 2009.

Tanaka M. Inactivation of the central thalamus delays self-timed saccades. Nat Neurosci 9: 20-22, 2006.

Tanaka M. Cognitive signals in the primate motor thalamus predict saccade timing. J Neurosci 27: 12109-12118, 2007.

Tanji J, Evarts EV. Anticipatory activity of motor cortex neurons in relation to direction of an intended movement. J Neurophysiol 39: 1062-1068, 1976.

Thach WT. Timing of activity in cerebellar dentate nucleus and cerebral motor cortex during prompt volitional movement. Brain Res 88: 233-241, 1975. 
Thach WT. Correlation of neural discharge with pattern and force of muscular activity, joint position, and direction of intended next movement in motor cortex and cerebellum. J Neurophysiol 41: 654-676, 1978.

Thach WT. On the mechanism of cerebellar contributions to cognition. Cerebellum 6: 163-167, 2007.

Thomas NW, Paré M. Temporal processing of saccade targets in parietal cortex area LIP during visual search. J Neurophysiol 97: 942-947, 2007.

van Kan PL, Houk JC, Gibson AR. Output organization of intermediate cerebellum of the monkey. J Neurophysiol 69: 57-73, 1993.

Weinrich M, Wise SP, Mauritz KH. A neurophysiological study of the premotor cortex in the rhesus monkey. Brain 107: 385-414, 1984.

Wiesendanger R, Wiesendanger M. Cerebello-cortical linkage in the monkey as revealed by transcellular labeling with the lectin wheat germ agglu- tinin conjugated to the marker horseradish peroxidase. Exp Brain Res 59: 105-117, 1985.

Wurtz RH, Sommer MA, Paré M, Ferraina S. Signal transformations from cerebral cortex to superior colliculus for the generation of saccades. Vision Res 41: 3399-3412, 2001.

Xiong G, Hiramatsu T, Nagao S. Corticopontocerebellar pathway from the prearcuate region to hemispheric lobule VII of the cerebellum: an anterograde and retrograde tracing study in the monkey. Neurosci Lett 322: 173-176, 2002.

Yamamoto T, Yoshida K, Yoshikawa H, Kishimoto Y, Oka H. The medial dorsal nucleus is one of the thalamic relays of the cerebellocerebral responses to the frontal association cortex in the monkey: horseradish peroxidase and fluorescent dye double staining study. Brain Res 579: 315-320, 1992.

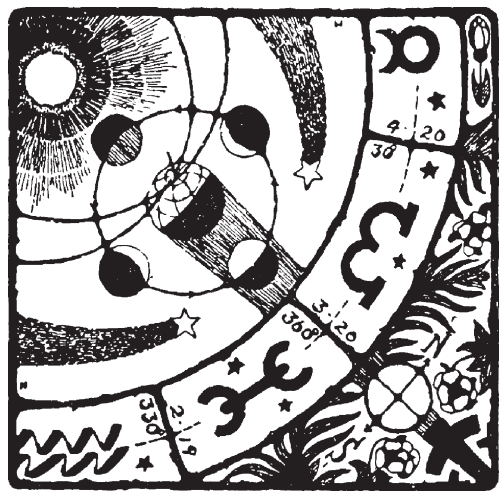

